\title{
Double Layer Dynamics in a Collisionless Magnetoplasma
}

lizuka, Satoru; Michelsen, Poul; Juul Rasmussen, Jens; Schrittwieser, Roman; Hatakeyama, Rikizo; Saeki, Koichi; Sato, Noriyoshi

\section{Publication date:}

1983

Document Version

Publisher's PDF, also known as Version of record

Link back to DTU Orbit

Citation (APA):

lizuka, S., Michelsen, P., Juul Rasmussen, J., Schrittwieser, R., Hatakeyama, R., Saeki, K., \& Sato, N. (1983). Double Layer Dynamics in a Collisionless Magnetoplasma. Risø National Laboratory. Risø-M No. 2414

\section{General rights}

Copyright and moral rights for the publications made accessible in the public portal are retained by the authors and/or other copyright owners and it is a condition of accessing publications that users recognise and abide by the legal requirements associated with these rights.

- Users may download and print one copy of any publication from the public portal for the purpose of private study or research.

- You may not further distribute the material or use it for any profit-making activity or commercial gain

- You may freely distribute the URL identifying the publication in the public portal

If you believe that this document breaches copyright please contact us providing details, and we will remove access to the work immediately and investigate your claim. 
RISO-M-2414

DOUBLE LAYER DYNAMICS IN A COLLISIONLESS MAGNETOPLASMA

Satoru IIZUKA ${ }^{x}$, Poul MICHELSEN, Jens JuUl RASMUSSEN, and ROMan SCHRITTWIESER ${ }^{\mathbf{X X}}$

Association EURATOM - Risø National Laboratory

Rikizo HATAKEYAMA, Koichi SAEKI, and Noriyoshi SATO

Department of Electronic Engineering

Tohuku University, 980 Sendai, Japan

Abstract. An experimental investigation of the dynamics of double layers is presented. The experiments are performed in a Q-machine plasma and the double layers are generated by applying a positive step potential to a cold collector plate terminating the plasma column. The double layer is created at the grounded plasma source just after the pulse is applied and it propagates towards the collector with a speed around the ion acoustic speed. When the collector is biased positively, large oscillations are obserced in the plasma current. These oscillations are found to be related to a recurring formation and propagation of a double layer. The period of the oscillations is determined by the propagation length of the double layer. The current is limited during the propagation of the double layer by a growing negative potential barrier formed on the low (continued on next page)

December 1983

Risø National Laboratory, DK-4000 Roskilde, Denmark 
potential tail. Similar phenomena appear when a potential difference is applied between two plasmas in a Q-machine with two sources. In this case a stationary double layer forms in the plasma column, but the low potential tail is subject to "back and forth" oscillations leading to large amplitude current oscillations.

UDC $533.95: 621.039 .61$

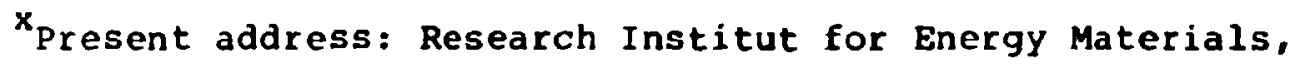
Yokohama National University, 240 Yokohama Japan

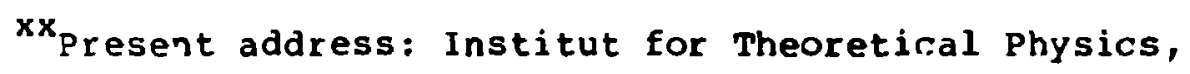
Innsbruck University. A-6020 Innsbruck, Austria

ISBN $87-550-0978-6$

ISSN $0418-6435$

Risø Repro 1984 
CONTENTS

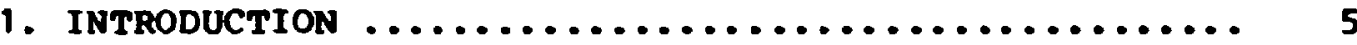

2. EXPERIMENTAL SET-UP AND METHODS $\ldots \ldots \ldots \ldots \ldots \ldots \ldots \ldots \ldots .10$

3. EXPERIMENTAL RESULTS $\ldots \ldots \ldots \ldots \ldots \ldots \ldots \ldots \ldots \ldots \ldots \ldots \ldots$

3.1. Single-ended operation ................... 13

3.2. Double-endeo operation .................. 20

4. DISCUSSIONS AND CONCLUSIONS $\ldots \ldots \ldots \ldots \ldots \ldots \ldots \ldots \ldots \ldots$

5. ACKNOWLEDGEMENTS $\ldots \ldots \ldots \ldots \ldots \ldots \ldots \ldots \ldots \ldots \ldots \ldots . \ldots . \ldots . \ldots$

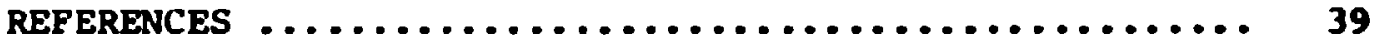

FIGURES $\ldots \ldots \ldots \ldots \ldots \ldots \ldots \ldots \ldots \ldots \ldots \ldots \ldots \ldots \ldots \ldots \ldots \ldots \ldots .45$ 



\section{INTRODUCTION}

Double layers $(\text { DLs })^{1,2}$ ) in plasmas have found increasing interest in recent years. They consist of localized potential jumps which cause acceleration and reflection of charged particles. Many experiments in various types of laboratory plasmas have clearly shown the existence of DLs (see e.g. Sato ${ }^{3}$ ) and references therein). In our previous work 4 ) we created a stationary DL in a magenetized plasma by applying a potential difference between two collisionless plasmas produced by surface ionization on hot plates in a Q-machine. The DL can attain an extremely large potential difference i.e. $e \Delta \phi / T_{e}-2 \times 10^{3}$, where $\Delta \phi$ is the applied potential. Similar results were obtained by Torvén5) in an experiment where the potential difference was applied betw. : n two discharge plasma sources. These were separated from the main plasma chanber by apertures in order to avoid ionization in the plasma by keeping the background pressure low. Strong DLs $\left(e \Delta \downarrow / T_{e}>1\right)$ can also be produced by injecting an electron beam into a plasma6-9) due to the space charge effects of the beam. Furthermore, DLs were observed in discharge plasmas, where they were found to form from the anode sheath10-11). However, in discharge plasmas with internal ionizations the DL potential is limited by the ionization potential of the background gas4,12). The formation of DLs were also investigated in several computer simulations (see e.g. Smith 13 ) and references tierein).

Most of the previous investigations have focussed on stationary DLs and comparatively few investigations were reported on their dynamics. The random generation of weak DLs with $\left.e \Delta \phi / T_{e}\right\}^{1}$, 
socalled ion acoustic DLs, in current carrying plasmas causes an effective electron scattering and gives rise to an anomalous resistivity as clearly demonstrated in recent computer simulations 14-15). Such structures seem to have been obserred recently in the auroral plasma 16$)$ and are thought to cause the acceleration of the precipitating electrons. Oscillating DLs were also proposed as a candidate for causing flickering auroras ${ }^{17)}$. Furthermore, recent observations of counterstreaming electron beams at an altitude of few earth radia above the auroral zone were explained in terms of fluctuating DLs $\left.{ }^{18}\right)$. Finall. it was found experimentally that the disruptions of DLs y-eld energetic tails in the ion distribution function and this is one of the important mechanisms for ion heating ${ }^{19}$ ). Therefore it is of ir-urtance to investigate the details of the stability and the dynamics of the DLs.

Propagating DLs were observed in the positive column in connection with current 1 imitations 20 ). However, becs:se of ionization of the background gas these DLs were disrupted and created randomly inhibiting a detailed study of the evolution. Moving DLs were also investigated in quiescent plasmas (e.g. Refs. 7, 21). In the experiment of Coakley and Hershkowitz 21 ) the stationary DL was made to propagate by abruptly increasing the plasma density in the region of the DL. The DL propagated from the region of high potential to the region of low potential with a velocity up to $3.5 \mathrm{C}_{\mathrm{s}}$ $\left(C_{s}\right.$ is the ion acoustic velocity) depending on the potential jump, and the evolution corresponds to that of a finite amplitude laminar electrostatic shock ${ }^{22}$ ). Leung et al.7) excited the moving DL by injecting a pulsed electron beam into the 
plasma and the DL propagated from the low potential region towards the high potential region with a velocity around $c_{s}$. They explained the propagation of the DL by the fact that only in the frame of reference moving with the $\mathrm{DL}$ the ion velocity did satisfy the requirements needed for the formation of a stationary DL. Somewhat similar arguments were advanced by Bergeron and Wright 23 ) and singh 24 ). In the results of Leung et $a 1.7)$ we notice a broad negative potential well on the low potential side of the DL, which moves together with the DL. The moving DL is also related to oscillations in the electron current, however, no detailed investigations of this relationship were presented. Singh and Schunk 25) have performed a detailed investigation of moving DLs using a numerical simulation and their results compare well with the observations of Leung et al.7) In particular they also find that the moving DL is accompanied by a negative potential well on the low potential tail of the DL.

The dynamics of moving DLs are also important for the formation of stationary DLs. Iizuka et al.8) investigated the formation of a DL by injecting a pulsed electron beam. The DL evolved from the Pierce instability26) generated at the beam inlet and moved towards the plasma center as it saturated. The stationary DL then appeared as the saturated state of the Pierce instability (see also Ref. 27). Note that this behaviour contrasts the observations of Leung et al.7) where a stationary DL was not formed when the electron beam was pulsed. In the experiments by sato et al.4) the formation process of the stationary DL was investigated by applying a potential pulse to one of the two plasma sources. 
The DL was initially formed near the grounded source but subsequently it moved towards the high potential plasma and eventually stcpped forming the stationary DL. The results of these investigations also suggested the existence of a small potential barrier formed on the low potential side of the moving DL limiting the current through the system. The stationary DLs were accompanied by low frequency potential oscillations localized on the low potential tail of the DLs. These fluctuations were responsible for an apparent broadening of the DL profile as obtained from time averaqed measurements and were especially pronounced sor lower DL-potentials ( $80 \mathrm{~V})$. Similar fluctuations in the DL profile were also found in other experiments (e.g. Ref. 10-11).

The present paper reports experimental investigations of the dynamical features of strong double layers in a nondischarge magnetized Q-machine plasma. The experiments are a continuation of the work on stationary DL formation reported in Ref. 4. Brief accounts of the results were reported previously 28,29). We have clarified some feature of moving DLs as appearing in our experiment by performing time resolved measurements 30 ) of the evolution of the plasma potential, the ion and electron saturation currents. Our investigations are devided into two main parts:

(i) Evolution of the DL when the Q-machine is operated with one source (single-ended) resembling the set up in Ref. 28. (ii) Evolution of the DL when the Q-machine is operated with two sources (double-ended) resembling the set up in Ref. 4.

In the first case the plasma column is terminated by a cold collector plate of variable bias. When a positive potential pulse 
is applied to the collector a DL is generated in front of the source and propagates towards the collector. The moving DL is accompanied by a negative potential barrier on the low potential tail which limits the current through the system. When a positive d.c. bias is applied to the collector it is known31) thst there appears an instability which shows many features of a standing ion acoustic wave. We clarify the mechanism of this instability and $f$ ind that it is caused by the dynamics of the negative potential barrier formed on the tail of the moving DL and an unstable sheath in front of the collector.

In the second case we investigate the details of the DL formation when one of the sources is pulsed 4 ). The behaviosr is similar to the one in the first case except that the DL, which originally forms at the grounded source and propagates towards the biased source, stops a finite distance from that source. We further examine the low frequency oscillations on the low potential tail of the stationary DL as found in Ref. 4. We find that these oscillations appear as a "back and forth" motion of the low potential foot point of the DL. Like in the single ended case the evolution of the DL is closely related to the dynamics of a negative potential barrier.

The plan of the paper is as follows: In Sec. 2 we describe the experimental set-up and the methods. The experimental results are presented in Sec. 3 both for the case with only one plasma source (3.1) and for the case with two plasma sources (3.2). Section 4 contains the discussions of the results and their relation to other works. 
2. EXPERIMENTAL SET-UP AND METHODS

The experiments were performed in the Riso Q-machine which was operated in either the double-ended or the single-ended mode 32). The set-up is shown schematically in Fig. 1. The plasma column is contained in a stainless steel vacuum vessel of $15 \mathrm{~cm}$ diameter. When the machine is operated double-ended the plasma is produced by surface ionization of cesium on two $3 \mathrm{~cm}$ - diameter hot tantalum plates (source $1\left(s_{1}\right)$ at $x=0$ and scurce $2\left(s_{2}\right)$ at $x=d=125 \mathrm{~cm})$. In the single-ended operation $S_{1}$ is replaced by a cold collector plate C (4 cm - diamater tantalum plate). In that case the length of the plasma column can be varied $110 \mathrm{~cm}<d<$ $120 \mathrm{~cm})$. The plasma is radially confined by a homogeneous magnesic field $B=0.4 \mathrm{~T}$. In all cases the machine is operated in the electron rich condition ${ }^{32)}$. The plasma density is $10^{7}-10^{9} \mathrm{~cm}^{-3}$, the electron and ion temperatures $T_{i} \approx T_{e}=0.2 \mathrm{eV}$, and the neutral background pressure is $10^{-4} \mathrm{~Pa}$ approximately. The mean free paths for collisions between charged particles and neutrals are much longer than $d$, and collisions are negligible. The plasma parameters are measured by an axial movable probe which functions as a usual Largmuir probe for measuring electron and ion saturation currents or as an electron emissive probe for measuring the plasma potential. The probe consists of an $8 \mathrm{~mm}$ long loop of $0.1 \mathrm{~mm}$ diameter tungsten wire. In the emissive operation the probe is heated either by a direct current or by a pulsed current which is off during the measuring time 30 ). The floating potential of the emissive probe is used for determining the plasma potential. To measure this potential accurately the probe resistance must be 
sufficiently large, i.e. much larger than the dynamical plasma impedance. This method has, however, a limited time resolution due to the stray capacitances and the cable capacitances. We emphasize that it is the dynamical plasma resistance we have to use in determining the time resolution and not the probe resistance as erroneously stated in Ref. 30. The plasma resistance is given by the slope of the probe characteristic at the floating potential. For the present case with relatively low density we found it to be around $100 \mathrm{kQ}$ giving a time resolution of around $0.1-1 \mathrm{msec}$. We therefore developed a method where the zero crossings of the probe currents, sampled at a given time, are detected. Then a comparatively low input resistance of $1 \mathrm{k} \Omega$ can be used. This enables us to obtain a time response of the emissive probe system better than a couple of $\mu \mathrm{s}$, which is fast enough to measure the DL-dynamics. Time resolved measurements are performed by means of a usual box-car sampling technique. This method and the emissive probe are described in details in Ref. 30 . Instead of using the analog zero-point detection as described in Ref. 30 the sampled probe signal is measured by an $A / D$-converter and monitored by a computer, which via a D/A-converter sweeps the probe voltage around the floating potential in order to keep track of the zerocrossing all the time. "Time-averaged" potential profiles are obtained by feeding the emissive probe signal directly into a xyrecorder through a large resistance (100 MQ).

To investigate the DL-dynamics we use three types of experimental set-ups which are shown in Fig. 1 . In the first setup the machine is operated in the single-ended mode with the source $S_{2}$ grounded together with the vacuum chamber. The plasma 
column is terminated by the cold collector plate $C$ with variable potential, $0 \mathrm{~V}<\mathrm{v}_{\mathrm{C}} \leqslant 100 \mathrm{~V}$. The collector is heated to around $700 \mathrm{~K}$ to prevent cesium coating, which changes the contact potential. First we measure the developments of the plasma potential, the electron, and ion saturation currents simultaneously, when a positive voltage pulse of short risetime $(<<1 \mu s)$ is applied to $C$ at $t=0$. Next we applied a positive d.c. potential to r. which gives rise to strong oscillations in the plasma potential and density. Simultaneous measurement of the plasma potential, the electron and ion saturation currents are performed during a period of the oscillations using the collector current as the triggering signal, and we clarified that the oscillations were related to the dynamics of a moving $D^{281}$.

In the second set-up we investigate the expansion of a plasma into the region between the grid $G$ and the positively biased collector C. The grid G (Fig. 1) of $4 \mathrm{~cm}$ diameter, made of stainless steel wires is inserted into the plasma column at a distance of $50 \mathrm{~cm}$ from $s_{2}(x=d-50 \mathrm{~cm})$. We chop the plasma flow into the region between the $G$ and $C$ by applying a pulse to $G$ rising from a negative bias, sufficient to reflect the plasma electron: from $\mathrm{s}_{2}$ and thereby stopping the plasma, to approximately the plasma potential. The plasma potential, the electron and ion saturation currents are measured during the plasma expansion.

In the third set-up the machine is operated in the doubleended mode and $C$ is replaced by the hot plate $s_{1}$. The plasma densities from $s_{1}$ and $s_{2}$ can be varied independently. We investigate the dynamics of DLs between two plasmas of dif- 
ferent potentials. This set-up is similar to the one used in Ref. 4. Quasi-stationary DLs are easily generated when a d.c.bias $V_{S 1}$ is applied to $s_{1}$ with respect to the grounded $i_{2}{ }^{4}$. Here we are interested in the dynamics of such quasi-stationary DLs. The measurements are performed in the same manner as in the cases of experiments in the single-ended mode.

\section{EXPERIMENTAL RESULTS}

\subsection{Single-ended operation}

We first investigate the DL-dynamics when the machine is operated with only one plasma source $s_{2}($ at $x=d$ ) and the plasma column is terminated by the cold collector C (at $x=0$ ) (Fig. 1). When a pulse with an amplitude of $V_{c}=30 \mathrm{~V}$ and a width of $700 \mu \mathrm{s}$ is app ied to $C$ at $t=0$ (Fig. 2a) the plasma potential evolves as shown in Fig. 2b. Just after $t=0$, the potential increases almost simultaneously in the whole column attaining a value almost equal to $V_{C}$. This is accompanied by an increase in the current to the collector $I_{c}$ (see Fig. 2c). (Immediately after $t=0$ the current is dominated by the effects of system stray capacities; this part is shown by a broken line). Then, proceeding from $\mathbf{s}_{\mathbf{2}}$ towards $C$ the potential begins to drop to zero, while Ic starts decreasing. Figure $3 b$ shows the temporal evolution of the spatial potential profile. For $t 275$ us we observe a luca?ized potential gradient of width 1 ess than $10 \mathrm{~cm}$ where nearly the entire potential drop is located. This potential profile has a DL-like form and is formed in front of source $s_{2}$. It moves towards the 
collector, C, with a speed of around $1.5 \times 10^{5} \mathrm{~cm} / \mathrm{s}$, which is almost equal to $3 C_{s}$, where $C_{s} \equiv \sqrt{2 T_{S} / M}$ is the sound speed. In the same period the current $I_{C}$ decreases (Fig. 2c). As the DL approaches the collector $C$ the potential jump and the electric field in the DL-region decreases. When it reaches the collector it becomes an ordinary sheath. The current $I_{c}$ then starts to incrsase again. By applying a shorter pulse (width $<500 \mu s$ ) we $f$ ind that when the pulse is shut off the DL continues to move with the same velocity with gradually decreasing potential jump and eventually disappearing while also the current $I_{c}$ vanishes.

The electron and ion saturation currents, $j_{e}$ and $j_{i}$, respectively, measured simultaneously with the potential measurements in Figs. $2 b$ and $3 b$ are shown in Fig. $2 d$ and $3 a$. We note that the saturation currents are not directly proportional to the plasma densities, since the beams in the plasma will contribute with a signal proportional to the beam flux and since the plasma potential is varying when the DL passes the probe. After $t=0 \mu s$ both $i_{e}$ and $j_{i}$ increase rapidly and reach maxima at $t=50 \mu s$ (Fig. 2d). Then they start decreasing gradually and after passing minima both currents increase rapidly again. Whereas the current maxima show almost no phase shift the time of the current minima shifts with $x$ (see also Fig. 4). Figure 3a shows that the saturation currents on the high potential side of the DL decrease as the DL propagates towards $C$. This is accompanied by a decrease in the collector current $I_{c}(F i g .2 c)$. However, as seen by the increase of the saturation currents on the low potential side, "new" plasma of higher density follows the DL. Fig. 4 shows an $x-t$ diagram of the $D L$ and the saturation currents, i.e. the 
position where the plasma potential is around zero just in front of the DL (broken lines), the positions of saturation current maxima obtained from Fig. 2d (full symbols), and the positions of saturation current minima obtained from both Figs. 2d and $3 a$ (open symbols) versus time. We observe that the ion saturation current has its minimum closer to the collector than the minimum of the electron saturation current. This indicates that the electrons are reflected at a position closer to the source than the ions, which suggests the existence of a negative potential dip on the low potential tail of the DL. The fact that je is very small on the high potential side of the DL also indicates the existence of such a dip. The collector current $I_{c}$ is mainly due to electrons that can overcome the dip, which thus acts as a current limiting mechanism. Note, however, that the potential of this dip is expected to be of the order of $T_{e} / e=0.2 \mathrm{~V}$ and is therefore hardly visible in Fig. 3b.

When a positive d.c. potential $v_{c}$ is applied to the collector low frequency oscillations $(1-10 \mathrm{kHz})$ appears spontaneously in the plasma31). Figure 5a shows the d.c. component of collector current $I_{c o}$ versus $V_{c}$. By increasing $V_{c}$ the current $I_{c o}$ first increases, then decreasees a little for $v_{c}>10 \mathrm{~V}$ and eventually saturates at an almost constant level for $v_{c} 230 \mathrm{~V}$. The frequency spectrum of the a.c. component of the collector current $I_{c}$ is shown in Fig. 5b. When $v_{c}$ is a few volts a broad low amplitude spectrum is seen and $T_{c}$ shows a random nonperiodic behaviour. The relative amplitude of these spikes in $I_{c}$ is rathe: low, i.e., less than 108 . For higher biases $\left(v_{c} 210 \mathrm{v}\right)$ the nonperiodic spikes evolve into regular coherent oscillations 
with a fixed period giving rise to clear peak in the spectrum. This corresponds to the current decrease in Fig. 5a. The anplitude of these oscillations increases with $v_{c}$ reaching more than 70 for $v_{c} \gtrsim 50 \mathrm{~V}$. Also the frequency is found to increase with $v_{c}$. The threshold between the random and coherent oscillations increases for increasing plasma column length d and density ${ }^{33}$ ). The profiles of the d.c.-components of the saturation currents $\overline{\mathbf{j}}_{\mathrm{e}}$ and $\overline{\mathbf{j}}_{\mathbf{i}}$ are plotted in Fig. $\mathbf{6}$ together with the d.c.-component of the plasma potential for different values of $v_{c}$. The saturation currents decrease along the column and show an overall decrease with increasing $v_{c} \cdot T$ becomes positive close to $\mathrm{C}$ and the region of positive reaches further out in the plasaa column as $v_{c}$ increases, but in all cases $\ll v_{c}$ except very close to $c$. For $v_{c} 20$ we observe the usual electron rich sheath in front of $C$.

In figs. 7 and 8 we show typical time resolved measurements of the saturation currents and the plasma potential, when the collector is biased positively $\left(v_{c}=72 \mathrm{~V}\right.$ i.e. well within the region of coherent oscillations). The evolution is quite similar to the pulsed case (Figs. 2-3). A moving DL propagates during the phase of the current decrease (Figs. $8 b$ and $7 b$ ) from the source $s_{2}$ to the collector $C$ with the speed 2-3 $C_{s}$. When the DL reaches $C$ it turns into a normal electron rich sheath, the current $I_{c}$ increases to its maximum in a rather short time ( $s 100 \mu \mathrm{s})$, and the potential, rises almost simultaneously along the whole plasma column (Fig. 7a) reaching a maximum value of around $30 \mathrm{~V}$ which is muc lower than $v_{c}(=72 \mathrm{~V})$. Thereafter begins to drop near $S_{2}$, the DL-like form reappears and starts to move towards $c$, while the current $I_{c}$ begins decreasing. This cycle 
repeats. The large anplitude oscillations appear also in the saturation currents as seen in Fig. $7 c$. The anplitude near $s_{2}$ is much larger than that near $C$. nowever, in Fig. 8a, which shows the spatial profiles of $j_{e}$ and $j_{i}$, we observe that $j_{e}$ and $j_{i}$ increase behind the $m \cdot$ ing $D L$, sinilarly as in the pulsed case Fig. 3. Therefore, the phase delay is caused by the expansion of a low-potential dense plasma with $=0 \mathrm{~V}$ produced at $S_{2}$ into a low density high-potential plasma which exists in the whole coluan at the time of current maximum. The plasma potential on the high potential side gradually decreases during the propagation. Figure 9 shows an $x-t$-diagran of the DL-evolution, i.e. the position where the plasma potential is zero just in front of the DL, obtained from Figs. Ta and $8 \mathrm{~b}$, versus time. The DL propagates towards $C$ with almost constant velocity $=1.5 \cdot 10^{5} \mathrm{~cm} / \mathrm{s}$ - $3 \mathrm{C}_{s}$ as were also found in the pulsed case (Fig. 4). This is the typical velocity for the plasma expansion in our device 34). When the DC approaches $C$ the velocity seens to increase. We note, however, that the DL-foot point is less well defined in this region. close to the collector does not become zero at all due to the formation of an ordinary electron rich sheath. The subsequent fast potential increase proceeds from $C$ to $S_{2}$ according to Pig. 9 with a speed higher than $2 \times 10^{6} \mathrm{~cm} / \mathrm{s}$. Thus the period of the oscillations is mainly determined by the time of flight of the DL towards $c$. We have performed similar measurements for different lengths $d$, densities and applied potentials obtaining essentially the same results. In fig. 10 the frequency $f$ of the oscillations is plotted versus inverse length $1 / d$ showing that f is roughly proportional to $1 / d$. Neglecting the time of the potential increase we find an averaged speed of the moving DL around $1.2 \cdot 10^{5} \mathrm{~cm} / \mathrm{s}\left(=(2-3) \mathrm{C}_{8}\right)$. 
For low collector biases, $v_{c} \leqslant 10 \mathrm{~V}$, where the instability evolves as random spiky oscillations, it is not possible to perform time resolved measurenents due to lack of coherence. However, simultaneous measurements with two probes at different axial positions indicate the sane behaviour as for the coherent oscillations, i.e., each spike corresponds to one cycle of the coherent oscrllation.

The measurements of the plasma potential are verified by recording the full probe characteristics sanpled at the given time for various positions. These measurenents further indicate the existence of an electron beam on the high potential side of the $\mathrm{DL}$, with a velocity determined by the potential jump.

More details of the fast build-up of the plasma potential, when a pulse is applied to the collector, are shown in Fig. 11, where the potential is obtained using the computer processing of the probe characteristics. Figure 11 a shows the potential pulse, $V_{c}$, applied to $C$ and the collector current $I_{c}$ on different time scales. In the lower figure with the expanded time scale we have subtracted the current pulse which is due to the stray capacities of the system. This is measured without plasma in the machine. The first part of the current trace is shown as a broken line to indicate the uncertainty of the real evolution. The current $I_{c}$ reaches a maximum at around 2 us and is rather constant for around $10 \mu \mathrm{s}$. The time of 3-4 $\mu \mathrm{s}$ is approximately equal to the .transit time of a thermal electron, while the time of 10 us roughly corresponds to one ion plasma period $(2 \pi /$ wpi $=20-6 \mu s$ for $n=$ $10^{7}-10^{8} \mathrm{~cm}^{-3}$ ). The plasma potential builds up almost uniformly 
along the column as shown in Fig. 11b. Possible oscillations and transients with a time scale shorter than a couple of $\mu$ s may not be detected, since such signals may be hidden in the capacitively coupled signal of the collector current. The time it cakes before - has become positive in the whole column ( 5 Hs corresponds to to the velocity of the fast build-up in the pulsed case as seen in Fig. 4. For the oscillatory case the build-up time of ti:e potential seems to be somewhat longer $=30 \mu s$. But for this case the accuracy of the measurements is smaller than in the pulsed case because the triggering signal is less coherent.

The measurements presented up to now show that the unstable oscillations are closely related to the dynamics of a moving DL followed by an expanding plasma. It is further indicated that the current $I_{c}$ is limited by a negative potential dip on the low potential tail of the DL. We looked for this potential barrier in measurements similar to Fig. 8 by increasing the sensitivity. However, because the ousillations were not sufficiently coherent and the barrier was expected to be of the order $\mathrm{T}_{\mathrm{e}} / \mathrm{e} \approx 0.2 \mathrm{~V}$ we could not find conclusive evidence for its existence. But to check our expectations, we performed an experimental simulation of the plasma expansion using the second set-up, i.e. the grid $G$ is inserted into the plasma column at $x=50 \mathrm{~cm}$ (in this case $d=100 \mathrm{~cm}$ ) (see Fig. 1). With the collector biased positively $\left(V_{c}=50 \mathrm{~V}\right)$ the grid potential $V_{G}$ is pulsed from - $40 \mathrm{~V}$ to around zero at $t=0$ as shown in Fig. 12a together with the evolution of the collector current, $I_{C}$. Thus, the plasma confined between $S_{2}$ and $G$ for $t \leqslant 0$ begins to diffuse towards $C$ at $t=0$. Figs. $12 b$ and $c$ show evolution of the plasma potential, $\phi$, and the saturation currents $j_{e}, j i$, 
respectively, in the region between $G$ and $C$. In the first cycle $t \leq 400 \mu s$, where the plasma simply expands we observe a clear negative potential dip in front of the plasma flow. For $t \leq 200 \mu s$ the depth of the dip grows until it reaches a value $o f=-0.4 \mathrm{~V}$ and the current $I_{C}$ is small. For $t_{22} 200$ Hs the depth decreases with the propagation and $I_{c}$ starts to increase. The dip disappears for $t=350$ us when it reaches the collector and $I_{c}$ attains its maximum. Then the potential builds up in the region between $G$ and $C$ and $a$ cyclic behaviour as in Fig. 8 starts. The moving DL together with the potential dip also apoears in the next cycle (400 $\mu$ s $<t<800 \mu s)$. By comparing Figs. $12 b$ and $c$ we find that the negative potential dip is moving in front of the expanding plasma. Therefore, the negative potontial dip is essential the same as the ambipolar potentiai in front of an expanding plasma. When the collector bias is negative ro potential dip is observed and the potential decreases monotonically in the region between the plasma front and the collector.

\subsection{Double-ended operation}

Next, we investigate the dynamics of the $D L$ when the machine is operated with two sources, $s_{1}$ (replacing $C$ ) and $s_{2}$ (see Fig. 1). In this case the length $d$ is $f i x e d, d=125 \mathrm{~cm}$. Double-layers are generated by applying a DC-potential $V_{S_{1}}=0-100 \mathrm{~V}$ to $s_{1}$ with respect to the grounded $s_{2}{ }^{4}$ ). Figure 13 shows typical time averaged profiles of the plasma potential and the saturation currents je,ji for $V_{S 1}=30 \mathrm{~V}$. The potential drops in the sheaths in front of $s_{1}$ and $s_{2}$ are relatively small (s few volts) and almost the entire potential $V_{S 1}$ appears as the potential drop $\Delta$ in the DL-region 
between the two plasmas. The currents $j_{e}$ and $j_{i}$ attain ainima in the DL-region and quite large gradients appear towards each plasa source. We have normalized $-j_{e}$ and $j_{i}$ to the same value at one end arked with the circle. On the low potential side of the $\mathrm{DL} j_{i}$ consists of a part $j_{i b}$ originating from the ion bean accelerated through the DL and a part $j_{i 2}$ coming from the ions created at $s_{2}$ and reflected at the DL, i.e. $j_{i}=j_{i b}+j_{i 2}$. while $j_{e}$ consi:zts of the electrons created at $s_{2}$ alone, $j_{e}=j_{e 2}$. Similar arguments can be applied on the high potential side of the DL. i.e. here $i_{e}=i_{e b}+$ $j_{e l}$ and $i_{i}=i_{i l}$. Since we have charge neutrality on both sides of the double layer $i . e . n_{i}=n_{e}$ we should expect $-j_{e}>i_{i}$ on the high potential side and $j_{i}>-j_{e}$ on the low potential side. because $j_{i}, j_{e}$ are measures of the fluxes. This is indeed also what is observed in Fig. 13. The strong gradients in $j_{i}$ and $j_{e}$ iaply gradients in the plasma density towards each source. More details of the stationary DL are presented in Ref. 4.

The dynamics of a propagating DL are investigated by applying a potential pulse to $s_{1}\left(V_{S 1}=25 \mathrm{~V}\right)$. The results are shown in Pig. 14. Just after applying $v_{S 1}$ at $t=0$ the plasma potential increases up to $V_{S 1}$ almost simultaneously in the whole coluan (Fiq. 14c) accompanied by an increase of the current Isi (Fig. 14b). The potential drop is then located at the sheath in front of $s_{2}$. After approximately 100 us the potential drop detaches from $S_{2}$ and a DL starts moving from $S_{2}$ towards $s_{1}$ simultaneously with a decrease in Is1. The evolution is quite similar to the observations in fig. 3. From profiles of the saturation current it may be seen that also in the present case the moving DL is followed by an expanding plasma from $s_{2}$ (zonfer also ref. 4 ). 
However, the DL stops at some position between $S_{1}$ and $s_{2}$. This is different from the evolution in the single-ended case (fig. 3 ). That position shifts along the coluan depending on the density ratio $N_{1} / N_{2}$, where $N_{1}$ and $N_{2}$ correspond to the density produced by $s_{1}$ and $s_{2}$, respectively. By increasing (decreasing) the ratio $N_{1} / N_{2}$ the final DL position moves toward $s_{2}\left(s_{1}\right)$. After the stop the DL is, however, subject to a "back and forth" oscillation of the low potential tail which is correlated to oscillations in the current $I_{51}$, as seen in Figs. 14b and $c$. The details of this evolution will be described in connection with Fig. 16. When the pulse is shut off at $t=1200 \mathrm{\mu s}$ the current drops to zero but the DL starts propagating further towards $s_{1}$ with decreasing potential drop. The characteristics of the evolution in Pig. 14 is independent of Vsl. The initial evolution of the moving DL in Fig. 14 was also observed in Ref. 4, where a pulsed bias on $S_{1}$ was used for investijating the $D L$ fornation.

Figure 15 shows the details of the potential profile on the low potential tail of the moving DL together with Isi (note that the conditions are not the same as in Fig. 14, i.e. $V_{S 1}=34 V$ ). After the $D L$ has detached from $s_{2}$ a negative potential dip appears on the low potential side of the $D L$ and follows it during the propagation. The dip grows to $t=-0.4 \mathrm{~V}$ at $t \leqslant 700 \mathrm{\mu s}$, while Is1 decreases to almost zero. Thus, the limitation of IsI is caused by the potential dip the depth of which $e / T_{e}=-2$ is large enough to reflect most of the electrons from $s_{2}$. The speed of the DL is almost constant $(=1.5 \cdot 105 \mathrm{~cm} / \mathrm{s})$ during this phase. for $t 2700 \mathrm{ks}$ the $\mathrm{DC}$ decelerates and eventually stops while the dip dissolves. Then the current $I_{S 1}$ increases again and a 
"stationary" DL is formed with oscillations on the low potentia. tail as observed in Fig. 14c. Similar oscillations appear when a d.c. potential $v_{S 1}$ is applied to $s_{1}$. These oscillations are correlated to oscillations in the plasma current $I_{S 1}$. Figure 16 shows an exanple of the evolution of the DL-profile within one period of the current oscillation which again is used as triggering signal. The Dr steepens during the current decrease, i.e. the low potential foot point woves towards $s_{1}$ (see fig. 16c). whereas the high potential edge is almost fixed. The maximum steepness is reached around the current minimum. At that instant the DL width is approximately $100 \lambda_{D}$. comparable with theoretical expectations. Thereafter the current increases within a relatively short time and the potential becomes broad again. This cycle repeats. The velocity of the foot point in the steepening phase is $\nabla=1.7 \cdot 10^{5} \mathrm{ca} / \mathrm{s}$ (Fig. 16c). The broadening, on the other hand, takes place on a much faster time scale i.e. $v>10^{6} \mathrm{~cm} / \mathrm{s}$. The period of oscillations is almost determined by the transit time of the foot point in the steepening phase. Accordingly, we find that the frequency is inversely proportional to the distance betweeen $s_{2}$ and the midpoint of the time averaged DL-profile, $L$, as shown in Pig. 16d. The evolution observed in Pig. 16 is guite similar to the single-ended case, if we consider the high potential plasma a virtual collector.

Figure 17 shows the periodic behaviour of the electron saturation current, je together with the corresponding averaged potential profile (for $V_{S 1}=30 \mathrm{~V}$ ). We observe a phase change along the coluan only in the region where the DL propagates, i.e. $\times 285 \mathrm{~cm}$. No phase change is detected on the high potential side of the DL 
where $j_{e}$ is in the phase with $I_{s 1}$. Thus, the phase delay again shows the expansion of the low potential plasma as was also observed in the single-erded case.

\section{DISCUSSION AND CONCLUSIONS}

In the present work we investigated the dynanical features of moving DLs in a magnetized fully ionized plasma in a Q-machine. Results from both the case where the machine was operated with only one source and the case where the machine was operated with two sources were presented. The properties are essentially the same for the two cases and we shall only discuss the evolution in the single source case in detail using these results to explain the evolution in the double source case.

Discussing the results obtained when the machine was operated with one source and a positively biased cold collector for terminating the plasma column, we note that there are two phases in the evolution of the instability, namely the moving DL during a current limitation and the fast increase of the potential in the whole column, when the DL reaches the collector. Even though the interest in moving DLs has been strongly increasing recently and they have been ohserved both in experiments $7,20,21$ ) and in simulations $13,24,25)$ no throughout theoretical explanation has been given of the dynamical evolution of strong DLs. Rather one has applied the stationary description in the frame of refesance moving with the UL explaining the motion as being caused by 
current imbalance (e.g. 23-25). In our experiment it is quite natural that a current imbalance would excist tcross the DL if it was stationary, since the density of the high-potential residual plasma is much lower than the density of the low potential plasma formed at the source. Thus the ion current $I_{i}$ through the DL cannot balance the electron current in a stationary state to satisfy the Langmuir condition $I_{e} / I_{i}=\sqrt{m_{i} / m_{e}}$ and the $D L$ will move towards the collector. The velocity of the DL is found to be $=1.5 \cdot 10^{5} \mathrm{~cm} / \mathrm{s}=3 \mathrm{C}_{\mathrm{s}}$, this is a typical flow velocity in the Q-machine 34$)$ where ions are accelerated through the electron rich sheath in front of the source. From Fig. 12 we see that the moving DL separates a high potential low density plasma from an expanding low potential high density plasma produced at the plasma source. During the ol propagation the current through the system is limited. This is caused by a negative potencial dip that forms on the low potential tail of the DL. The depth of the dip grows to be on the order of the electron thermal energy and becomes an effective barrier decreasing the number of electrons passing through the system. Thus, the dip acts like a thermal barrier. The formation of the negative potential dip resembles the ambipolar potential formed in front of an expanding plasma. The potential dip can exist consistently only in the moving frame with a velocity larger than or comparable to the ion flow velocity. In a stationary frame the ions will cancel the negative space charge connected with the dip, because the potential of the adjacent DL is much larger than $T_{e} / e$ (see e.g. Ref. 35). Thus when the DL slows down and finally stops at the collector the dip is filled in with ions and a usual electron rich sheath forms. Then the electrons are free to flow and the current increases rapidly. 
This is the start of phase two of the evolution. The rapid current increase is apparently connected with a sheath instability and the plasma potential rises in the whole column within a very short time as compared to the time of the DL propagation. The rime of the potential build-up is so short that the ions have no time to react appreciably and the change of potential is caused Jy an electron rearrangement only. A tentative explanation of this sheath instability is proposed as follows: when the electron rich sheath is formed at $c$ the electrons produced at $s_{2}$ are not accelerated and they do not automatically satisfy the Bohm criterion ${ }^{1)}$ when entering the sheath. Therefore, a presheath will form to accelerate the electrons to the necessary velocity. We point out that such a presheath cannot be expected to become stationary, but the edge of the presheath, separating it from the neutral plasma will propagate away from the main sheath with a velocity around the electron thermal velocity. Such a behaviour was found in connection with the evoloution of an ion rich sheath 36 ), where a quasi-neutral expansion region takes care of the self consistent ion acceleration. The expansion region, which corresponds to the presl:ath, propagates with the ion-acoustic speed. This evolution was re sently confirmed in a numerical simulation 37). In a semiinfinite plasma the presheath will ultimately have an "infinite" extent and cannot be distinguished in the plasma. But if the plasma is of finite extent the edge of the presheath will reach the boundary and some electron rearrangements will take place. In the present case we thus expect the edge of the presheath to propagate towards $s_{2}$ with a velocity around the electron thermal velocity. During the propagation the main sheath is stable since the electrons 
entering it satisfy the Bohm criterion. When the presheath reaches the electron rich sheath in front of $s_{2}$ this is modified and some electron rearrangements wili take place which causes the current increase and may cause the disruption of the sheath. Although the time scales involved in these arguments are consistent with our observations, more investigations are necessary to clarify the role played by such a presheath evolution.

Some details of this fast potential build-up may be gained from the initial evolution in the case where the collector bias is pulsed (see Figs. 2-4 and 11) suggesting the following evolution (see also the related investigations in Refs. 38-40): Just after the pulse is applied the electrons are quickly drawn out of the plasma and the current increases to its maximum value within around $2 \mu \mathrm{s}$. The ions will not respond so quickly thus the removal of the electrons will leave a positive space charge in the column and a positive plasma potential will build-up as seen in Fig. 11. We should stress here that the time resolution $(=1 \mu s)$ of our measuring system is just marginal for these investigations and possible oscillations with a shorter time scale will not be detected. Further due to this limitation the potential build-up might be somewhat faster than indicated in Fig. 11b. The new state with a positive plasma potential in the whole column cannot persist when the ions start to react after few ion plasma periods. They are returned to the scurce and the potential profile starts to relax from the source as the density of the high-potential plasma decreases. Consequently the current is observed to decrease after around $10 \mu s\left(\approx 4 \pi / \omega_{p i}\right)$ as seen in Fig. 11 a. 
Ultimately a DL detaches from the source and moves towards the collector. The cyclic behaviour is then started.

The investigations of Clark and Hamberger ${ }^{39)}$ performed in a set-up similar to ours complements in some respect our investigations. They examined the very initial behaviour of the plasma parameters when a pulse, so short $(2 \mu s)$ that the ions were not appreciably affected, was applied to the collector. Their results indicate that electron plasma wave pulses are responsible for establishing the plasma current and building up the potential in the column. Furthermore they found that the current is saturated because of an anomalous resistivity due to the build-up of a twostream turbulence. They note that the current was found to decay rapidly after 3-4 $\mu$ s if the applied pulse is prolonged, similarly to our observations (their density was somewhat higher than ours and they used potassium ions). Thus the current limitation due to the moving DL is far more effective than the limitation due to the anomalous resistivity of the two-stream turbulence. In the work by Schrittwieser and Rasmussen 40 ) the potential pulse was applied to a grid collector in a single-ended Q-machine and the details of the plasma response were investigated by measuring the ion saturation current. Four different successive pulses were observed initially. An electron plasma wave pulse followed by an ion burst which was overtaken by a highly supersonic positive ion pulse, all propagating from the grid to the source. Thereafter an ion rarefaction pulse propagated from the source towards the grid. The two latter signals correspond to the maxima and minima, respectively, of the saturation currents in Fig. 2d. The appearance of the burst with a velocity corre- 
sponding to the full amplitude cf the applied pulse was taken as an evidence that the potential did not $r$ ise in the whole column on a time scale too fast for the ions to follow. Rather it seemed that the burst, originating from ions initially trapped in the ion rich sheath around the grid (the potential pulse started from a negative potential), delayed the potential build-up. This build-up was assumed to be connected with the supersonic ion pulse. When the potential pulse started from $0 \mathrm{~V}$, as in the present case, no burst was observed and the supersonic pulse was less delayed. The evolution of this pulse, and thereby the potential build-up, was tentatively explained as a result of a transient Buneman instability giving a characteristic time for the build-up which is on order of what we have observed. This explanation is somewhat similar to the one used by clark and Hamberger ${ }^{39)}$ under unstable conditions.

Our experimental set-up (in the single-ended case) has some similarities with the thermionic converter or the plasma diode which has been extensively investigated during the last two decades (see e.g. Ref. 41, references therin and Ref. 42). However, in this device the distance between the hot plasma source and the collector is in general very short ( $x 100$ Debye lengths) as compared to our set-up (further in the converter the plasma is usually not magnetized). When the collector is biased positively large-amplitude oscillations appear in the current through the device. Burger 43) investigated the mechanism of these current oscillations using a numerical simulation and the evolution of the current and the plasma potential is indeed very similar to our results (e.g. Fig. 8 ), i.e. the current oscillations are related to the dynamics of a potential dip limiting 
the current and an unstable sheath. The mechanism of this instability is also quite similar to the Gunn-diode oscillations, where the DL propagates in a semiconductor 44 ). The electrons in the solid get energy from the electric field and are excited to a high energy level where the mobility of the electrons becomes small. The transition between the low and high energy levels causes a negative resistance in the system and the instability appears. The electrons with high and low mobilities in the Gunndiode correspond to the free beam electrons and those reflected from the barrier, respectively, in our case.

Furthermore our results provide a detailed understanding of the mechanism of the current driven instability excited by a positively biased collector-grid or -plate. In the previous experiments in Q-machine plasmas 31 ) this instability was considered to appear as a half standing ion acoustic wave without detailed investigations of its dynamics. Referring to our observations the instability should rather be termed a "potential relaxation instability". A somewhat similar explanation was previously suggested by Rynn 45 ). This type of behaviour of an instability excited by the current drawn to a positively biased collector is not an artefact of our experimental apparatus, but has recently been observed also in a discharge plasma46) (a double plasma device with $T_{e} \gg T_{\dot{i}}$ ). In that connectic" we believe that the socalled instantaneous feedback mechanism invoked in explaining the behaviour of a standing wave instability excited by a current in similar experiments ${ }^{47}$ is explained by the fast potential build-up. It should also be mentioned that Buchelnikova and Salimov 48 ) explained a similar instability in a Q-machine plasma as relaxation oscillations. 
However, the fundamental instability excited in their experiment, where the current was drawn to a target consisting of small circular seqments isolated from one another, was the current driven ion cyclotron instability of relatively hiqh frequency. This instability caused an enhanced radial diffusion, which in turn was thought to quench the instability and then new plasma filled the machine. The observed low frequency instability was then explained as relaxation oscillations. We enphasize that we have not observed oscillations during the build-up phase, and in particular no oscillations with frequencies around the ion cyclotron frequency. This is in keeping with recent observations by Schrittwieser 49), who showed, using an anullar collector, that the ion cyclotron instability was only excited when the collector diameter was sufficiently small ( $s$ a couple of ion Larmor radia), while a collector of larger diameter preferentially leads to the excitation of the low frequency "potential relaxation instabilityn.

In general the preceeding discussion shows that experimentally it seems hardly possible to obtain the equilibrium potential distribution 50 ) in a single-ended Q-machine with a positively biased collector, since it seems to be unstable. However, in a recent experiment Popa et al.51) succeeded in suppressing the instability when the applied bias exceeded a certain threshold valis. The experiments were performed in a single-ended Q-machine with a very short plasma column $(=10 \mathrm{~cm}$ ) and it was suggested that in the stabilized state the potential distribution is described by the equilibrium model 50) although the plasma potential was not measured explicitly (see also Ref. 52). The 
results of Popa et al.51) are in qualitative agreement with the predictions of Rurnetsov and Énder 42 ), who have calculated the stability regimes of the plasma diode. Furthermore they have theoretically examined the evolution of the instability and found results qualitatively in agreement with our observations. We should emphasize, however, that for usual Q-machine operation with a plasma length of -1 the bias necessary for stable operation will be in the range of several kV as extrapolated from the results of Ref. 42 and thus far outside the normal operation range.

The evolution of the DLs in double-ended operation was quite similar to the behaviour in the single-ended case if we inagined the high potential edge of the stationary DL as a virtual collector. Note that this edge is fixed when a d.c. bias is applied to $S_{1}$. The oscillations in the DL profile, which were observed to appear as "back and forth" motion of the low potential foot point of the DL (Fig. 16) are especially pronounced for relative small values of $v_{S 1}(s 80 \mathrm{v})^{4)}$ and they may to some extent be suppressed by adjusting the ratio of the densities supplied from the two sources 4 ). In the case shown in Pig. 16 we chose the parameters to maximize the fluctuation amplitude to facilitate the measurements. That the high potential edge of the DL really is $f$ ixed under almost all conditions may be related to the fact that the ions produced at $s_{1}$, are accelerated through the electron rich sheath and thus attain a drift velocity sufficient to satisfy the Bohm criterion') when entering the DL. On the low potential side, however, we expect similar effects as in the single-ended case, thus the periodic disruption of the DL may be related to the dynamics of the presheath. 
We investigated the formation of the stationary DL by applying a potential pulse to the source $S_{1}$ (Figs. 14, 15). The evolution of the plasma potential is also here similar to the evolution in the single-ended case when the collector bias is pulsed (Figs. 2-4) except that the density of the high potential plasma is not decreasing 4 ) and the potential jump of the moving DL is constant, almost equal to the applied voltage. The DL is expected to move because of a current imballance, thus the DL will stop when there is current balance, i.e. when the currents through the DL satisfy the Langmuir condition $I_{e} / I_{i}=\sqrt{m_{i} / m_{e}}$. In order that this condition is satisfied at one point in the plasma column, we have to assume small radial losses, which will cause slight gradients in the densities $N_{1}$ and $N_{2}$ of the plasmas produced at $s_{1}$ and $s_{2}$, respectively. If $\mathrm{N}_{1}$ and $\mathrm{N}_{2}$ were constant outside the DL-region the Langmuir condition would either be satisfied at every point in the column or not at all. Such an axial variation of $N_{1}$ and $\mathbf{N}_{2}$ also explains that the position of the stationary DL can be shifted towards $S_{1}\left(S_{2}\right)$ by increasing $N_{2} / N_{1}\left(N_{1} / N_{2}\right)$. Similar arguments were used by Torvén5) in explaining the position of the DL in his experiment.

In comparing our results with other investigations of moving DLs we first note that our observations in the single-ended case are in close agreement with the results of Leung et al 7) in spite of the apparent differences in experimental set-ups, and plasma parameters. In particlular they also found a negative potential barrier accompanying the moving $D L$ and limiting the current. They further observed recurring formation of the $D L$ and the frequency of this recurring formation was determined 
by the travelling time of the moving DL. However, they did not investigate the evolution of the density during the DL propagation as was attempted in the present work by measuring the saturation currents to a Langmuir probe. Although these currents are not directly a measure of the plasma density as pointed out in connection with Figs. $2 a$ and $3 a$, some conclusions concerning the density variation can be drawn from them. For instance it is evident from figures like $2,3,7,8$, that the DL woves along with a strong density depression, and that the DL on the low potential side is followed by an expanding plasma front. These observations also hold true for the double-ended case. The experiment of the Coakley and Hershkowitz ${ }^{21}$, on the other hand, concerns a quite different problem in that the DL here was moving towards the low potential side and thus takes form of a conpressional shock. In the positive column20) a moving DL forming at a density discontinuity was responsible for a strong current limitation. An even more drastic effect connected with the formation of DLs in current carrying discharge plasmas was observed in the turbulent heating experiments of Kalinin et al.53). Here a rapid decrease and redistribution of the plasma current was accompanied by the formation of a DL-like potential structure and a termination of the heating. In these experiments 20,53) it was not possible to study the details of the DL-formation because DLs were formed and disrupted randomly due to the effects of internal ionization.

Many features of moving DLs have been clarified by using numerical simulations (see e.q. 13, 24, 25, 54). The results of Singh and Schunk25) showed that the picture of the DL motion 
based on the current imbalance as discussed above is only a part of a much more complex picture involving such processes as plasma heating, evacuation of the plasma from the high potential side forming of a density gradient across the DL, the propagation of a density front with nearly the ion acoustic speed following the low potential tail of the DL, and the fornation of a negative potential dip limiting the current. They observed a periodical formation and motion of the DL associated with a recurring interruption of the electron and ion currents through the system. The time period of these recurring formations were propotional to the system length as also found in our experiment. However, many of these features and their inter-relations still need theoretical clarification. We note that although the simulation of singh and Schunk 25) do not model the details of our set-up especially with respect to boundary conditions our results compare remarkably well with the simulation results. In the simulation model a fixed potential was applied across the systen, as in our case, but the electrons and ions were injected from the low and high potential side, respectively, with prescribed velocities, whereas in our experiment these velocities are determined self-consistently by the sheaths in front of the sources. These differences seem at least to qive rise to a somewhat different formation mechanism when the DL is reformed near the source. In the sinulations (Sinah 25), it seems that a positive potential pulse with the qualitative features of an "electron hole-55) plays a decisire role in the initial phase of the DL formation. which was also seen in other simulations 54 ). This pulse is formed near the low potential boundary and moves in the direction of the electron drift. It is formed because electrons accelerated in the applied 
electric field are removed faster than they can be supplied by the plasa reservoir at that boundary and because the ions will not react on this time scale 54 ). We do not observe such a pulse in our experiment naybe because our time resolution is too 10 . However. the echanisa we discussed in connection with the fast potential rise was based on sonewhat sinular arquents. As also concluded in Ref. 25 (see also Ref. 13 for a thorough discussion) the boundary conditions have a large influence on the detailed dynanics of DLs. While the overall picture of the DL wotion as observed in this work and in Refs. 7, 20, 24 and 25 seens to be quite general. In understanding all the features observed in this work it would be desirable to perform a sinulation using the physical boundary conditions as attempted by Burger ${ }^{43)}$, and a system length comparable to the physical system. In particular it could be of great interest to see how such features as the formation and evolution of presheaths would influence the DL formation and stability.

Recently Silevitch 17 ) (see also Ref. 56) exanined the stability of a DL with special attention to the auroral DL. He was quided by the model of curtent oscillations in the plasma diode based on the results of Burger 43 to suggest a mechanism for the periodic disruptions of DLs similar to our observations. He showed by applying a variational method that a realistic analytical model for a DL behave like a negative dynamical resistance on a fast time scale of the electron motion. The effects of the neqative resistance would lead to periofic disruptions of the DL with a frequency of around $10 \mathrm{~Hz}$ for the auroral DL-case, and the fluctuating DL was proposed as a candidate for flickering auroras ${ }^{17}$ ). 
This frequency was determined by the ion transit time through the DL-reqion.

Finally we will briefly discuss our findings in connection with the observations of ion-acoustic DLs in computer sinulations (5). It has been suggested in many independent works57-60) that the existence of a negative potential dip in the form of a solitary structure in a current carrying plasea leads to the formation of the ionacoustic double layers observed in the sinulations $13,14,15$. These DLs are very weak with a potential jump $\Delta \& \mathrm{~T}_{e} / e$. The negative potential dip observed in our experiment accompanying the propagating strong $D L, \Delta \uparrow \gg T_{e} / e$, acts in a similar way as the abovementioned solitary structure with respect to current limitation. But we should emphasize that it is of different origin. In our case the dip is formed on the low potential tail of the moving DL in front of an expanding plasma. We arqued that this dip cannot exist in a stationary frame because it accompanies a strong DL $\Delta \bullet \gg T_{e} / e$, and will be cancelled by inflowing ions when it stops. The potential dip connected with the ion acoustic DL, is believed to be created by the ion acoustic turbulence in the current carrying plasma (5,58-60), and can exist in a stationary frame where ion trapping results in the formation of an ion hole58,61). The scenario of the formation of an ion acoustice DL preceeded by the development of a solitary ion hole $57-60$ ) is clearly revealed in the numerical simulations $14,15,59,60)$. The ion acoustic DLs are not stationary beyond the ion inertia time scale and they are found to decay by emitting ion acoustic solitary waves (potential humps). In the simulations with long systems many DLs were found to form in series ${ }^{15}$ ), and the number increased with the 
systen length. The recent observations of Temerin et al.16) suggests that many of these acoustic Dus in series form in the auroral plasma and that the precipitating electrons gain their energy of few $k V$ by passing through this series of DLs all of which are relatively weak.

Also experimentally it was observed that multiple double layers could form if the system was sufficiently long62,63). The DLs were generated by applying a potential difference between two plasma sources. It should be stressed that the observed DLs all have $\Delta \downarrow>\mathrm{T}_{\mathrm{e}} / \mathrm{e}$ and cannot be described as ion acoustic DLs. Chan and Herskowitz62) found a transition from a single DL to multiple DLs when the scale parameter $\varepsilon=\left(\lambda_{D} / L\right)^{2}$ (L is the system length) was smaller than 10-4. For the experiment of Hollenstein et al.63) $\varepsilon \leq 10^{-6}$. In our experiments we have not observed the formation of multiple DLs in any case even when extremely high DLs were formed $\left.\Delta,=400 \mathrm{~V}^{4}\right)$ although $\varepsilon \leq 10^{-6}$. Both the cited experiment $\$ 62,63)$, however, were performed in plasmas with internal ionizations and it is quite probable that this is the main cause for the multiple DL structure since it seems that the multiple DLs are formed when the applied potential exceeds the ionization potential. 


\section{ACKNOWLEDGENENTS}

We thank B. Reher and M. Nielsen for their technical assistance. The work was carried out while two of the authors (S.I. and R.S.) were visiting the Rise National Laboratory. They acknowledge the supports of this institution and are thankful for the cordial hospitality of their collaborators. This work was partially supported by Grant-in-aid for the Sakkokai Foundation (Japan) and the Fonds zur Förderung der Wissenschaftlichen Forschung (Austria) under Grant No. S-18/02.

\section{REFERENCES}

1) P. Carlquist: Wave Instabilities in Space Plasmas, ed. P.J. Palmad. so and R. Papartopoulos (Reidel, Dordrecht, 1979) P. 83; S. Torven: ibid. P. 109; L.P. Block: Astrophys. Space Sci. 55 (1978) 59 .

2) Proceedings of the Symposium on Plasma nouble Layers, Risa National laboratory, June 16-18, 1982 (ed. P. Michelsen and J. Juul Rasmussen) Risa R-472.

3) N. Sato: in Ref. 2 pp. 116-14n.

4) N. Sato, R. Hatekeyama, S. Iizuka, T. Mieno, K. Saeki, J. Juul Rasmussen, and P. Michelsen: Phys. Rev. Lett. 46 (1981) 1330; N. Sato, R. Hatekeyama, S. Iizuka, T. Mieno, K. Saeki, J, Juul Rasmussen, P. Michelsen, and R. Schrittwieser: J. Phys. Soc. Japan 52 (1983) 875. 
5) S. Torvén: J. Phys. D.: App. Phys. 15 (1982) 1943.

6) P. Coakley and N. Hershkowitz: Phys. Fluids 22 (1979) 1171 .

7) P. Leung, A.Y. Wong, and R.H. Quon: Phys. Fluids 23 (1980) 992.

8) S. Iizuka, K. Saeki, N. Sato, and Y. Hatta: Phys. Rev. Lett. 43 ( 1979$) 1404$.

9) K.D. Baker, N. Sinqh, L.P. Block, R. Kist, W. Kampa, and H. Thiemann: J. Plasma Phys. 26 (1981) 1 .

10) S. Torven and D. Anderson: J. Phys. D: Appl. Phys. 12 (1979) 717.

11) S. Torven and L. Lindherg: J. Phys. D.: Appl. Phys. 13 (1980) 2285.

12) S. Iizuka, P. Michelsen, J. Juul Rasmussen, R. Schrittwieser, R. Hatakeyama, K. Saeki, and N. Sato: in Proceedings of XVth Int. Conf. on Phenomena in Ionized Gases, Minsk, 1981, part 1. p. 501-502.

13) R.A. Smith: Physica Scripta T2 (1982) 238.

14) J.S. DeGroot, C. Barnes, A.E. Walstead, and O. Buneman: Phys. Rev. Lett. 38 (1977) 1283 .

15) T. Sato and H. Okuda: Phys. Rev. Lett. $\underline{44}$ (1980) 740; J. Geophys. Res. 86 (1981) 3357. H. Okuda and M. AshourAbdalla: Phys. Fluids 25 (1982) 1564 .

16) M. Temerir,, K. Cerny, w. Lotko, and F.S. Mozer: Phys. Rev. Lett. $\underline{48}(1982) 1175$.

17) M.B. Silevitch: J. Geophys. Res. $\underline{86}$ (1981) 3573.

18) R.D. Sharp, E.G. Shelley, R.G. Johnson, and A.G. Ghielmetti: J. Geophys. Res. $\underline{85}$ (1980) 92.

19) K. Saeki, S. Iizuka, and N. Sato: Phys. Rev. Lett. $\underline{4 b}$ (1980) 1853. 
20) E.I. Lutsenko, N.D. Sereda, and L.M. Kontsevoi: Zh. Tekh. Fiz. 45 (1975) 789. [Sov. Phys. Tech. Phys. 20 (1975) 498] and Fiz. Plasmy 2 (1976), 72 [Sov. J. Plasma Phys. 2 (1976) 39].

21) P.G. Coakley and N. Hershkowitz: Phys. Lett. 83A (1981) 131.

22) D. Montgomery and G. Joyce: J. Plasma Phys. 3 (1969) 1.

23) K.D. Bergeron and T.P. Wright: Phys. Fluids 21 (1978) 1578.

24) N. Singh: Phys. Lett. 75A (1979) 69.

25) N. Singh and R.W. Schunk: J. Geophys. Res. 87 (1982) 3561;

N. Singh: Plasma Phys. 24 (1982) 639.

26) J.R. Pierce: J. Appl. Phys. 15 (1944) 721 .

27) M.V. Nezlin: Fiz. Plasmy I (1981) 1048. [Sov. J. Plasma Phys. I (1981) 575].

28) S. Iizuka, P. Michelsen, J. Juul Rasmussen, R. Schrittwieser, R. Hatakeyama, K. Saeki, and N. Sato: Phys. Rev. Lett. $4 \mathrm{~B}$ $(1982) 145$.

29) S. Iizuka, P. Michelsen, J. Juul Rasmussen, R. Schrittwieser, R. Hatakeyama, K. Saeki, and N. Sato: Proc. Int. Conf. Plasma Phys. June 9-15 (1982) Goteborg, Sweden. Contributed papers p. 134; In Ref. 2 pp. 199-204.

30) S. Iizuka, P. Michelsen, J. Juul kasmussen, R. Schrittwieser, R. Hatakeyama, K. Saeki, and S. Sato: J. Phys. E: Sci. Instrum. 14 (1981) 1291; Plasma Res. Rep. Tohoku University THUP-2 (1981).

$31)$ N. Sato, G. Popa, F. Mark, E. Mravlag, and R. Schrittwieser: Phys. Fluids 19 (1976) 7n; R. Schrittwieser: Phys, Lett. 65A (1978) 235; P. Michelsen, H.L. Pecseli, J. Juul Rasmussen, and R. Schrittwieser: Plasma Phys. 21 (1979) 61.

32) R.W. Motley: "O-Machines" (Academic, New York, 1975). 
33) R. Schrittwieser, E. Mark, and S. Kuhn: J. Physique 40 Supp. au No. 7 (1979) C7 - 581; R. Schrittwieser: Phys. Lett. 95A (1983) 162.

34) H.K. Andersen, N. D'Angelo, P. Michelsen, and P. Nielsen: Phys. Fluids 11 (1968) 606.

35) M.T.C. Fang, D.A. Fraser, and J.E. Allen: Brit. J. Appl. Phys. $\underline{2}$ ( 1969$) 229$.

36) J.W. Cipolla and M.B. Silevitch: J. Plasma Phys. 25 (1981) 373.

37) 4. Skbelv, Univ. of Troms8, Norway: (private communication).

38) N. St. J. Braithwaite and J.E. Allen: J. Physique 40 Supp. au No. 7 (1979) C7-491; Int. J. Electronies $\underline{51}$ (1981) 637 .

39) W.H.M. Clark and S.M. Hamberqer: Plasma Phys. 21 (1979) 943.

40) R. Schrittwieser and J. Juul Rasmussen: Phys. Fluids 25 (1982) 48 .

41) C.K. Birdsall: in Ref. 2 pp. 84-89.

42) V.I. Kuznetsov and A. Ya. Énder: Zh. Tech. Fiz. 47 (1977) 2237 [Sov. Phys. Tech. Phys 22 (1977) 1295];

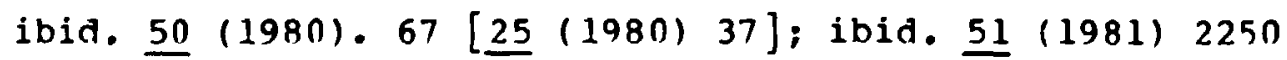
[26 (1981) 1326]; V.I. Babanin, I.N. Kolyshkin, V.I. Kuznetsov, A.S. Mustafaev, V.I. Sitnov, and A. Ya. Énder: ibid. 52 (1982) 1304 [ 27 (1982) 793].

$43)$ P. Burger: J. Appl. Phys. 36 (1965) 1938; w. Cutler and P. Burqer: ibid. 37 (1966) 2867 .

44) J.R. Gunn: Solid State Commun. 1 (1963) 88.

$45)$ N. Rynn: Phys. Fluids 9 (1966) 165.

46) H. Fujita, S. Yagura, F. Yamada, Y. Kawai, and N. Sato: in Ref. 2 pp. 2n9-212. 
47) H. Tanaca, A. Hirose, and M. Kogenei : Phys. Rev. 161 (1967) 94 .

$48)$ N.S. Buchelnikova and R.A. Salimov: Zh. Eksp. Teor. Fiz. 56 (1969) 1108. [Sov. Phys. - JETP 29 (1969) 595].

49) R. Schrittwieser: Phys. Fluids 26 (1983) 2250.

50) S. Kuhn: Plasma Phys. 21 (1979) 613; ibid. 23 (1981) 881 .

51) G. Popa, M. Santuloviciu, S. Kuhn, M. Dertl, and R. Schrittwieser: Phys. Lett. 87A (1982) 175.

52) S. Kuhn: Phys. Rev. Lett. 50 (1983) 217; S. Iizuka, P. Michelsen, J. Juul Rasmussen, R. Schrittwieser, R. Hatakeyama, K. Saeki, and N. Sato: ibid. 218.

53) Yu.G. Kalinin, D.N. Lin, L.I. Rudakov, V.D.Ryutov, and V.A. Skoryupin: Zh. Eksp. Teor. Fiz. $\underline{59}$ (1970) 1056 [Sov. Phys. JETP 32 (1971) 573].

$54)$ G. Joyce and R.F. Hubbard: J. Plasma Phys. 20 (1978) 391. R.F. Hubbard and G. Joyce: J. Geophys. Res. 84 (1979) 4297.

55) K. Saeki, P. Michelsen, H.L. Pécseli, and J. Juul Rasmussen: Phys. Rev. Lett. 42 (1979) 501 .

56) M.A. Raadu and M.B. Silevitch: in Ref. 2 pp. 60-64.

57) A. Hasegawa and T. Sato: Phys. Fluids 25 (1982) 632.

58) H. Schamel: in Ref. 2 pp. 13-39, Physica Scripta T2 (1982) 228.

59) K. Nishihara, H,. Sakagami, T. Taniuti, and A, Hasegawa: in Ref. 2 pp. 41-46.

60) G. Chanteur, I.C. Adam, R. Pellat, and A.S. Volokhitin: Phys. Fluids 26 (1983) 1584; in Proc. 1982 Int. Conf. Plasma Phys. Göteborg, Sweden, June 9-15, contributed papers p. 38.

61) H.L. Pécseli, R.J. Armstrong, and J. Trulsen: Phys. Lett. 81A (1981) 386; Ref. 2 pp. 47-54; Physica Scripta (1984) in press. 
62) C. Chan and N. Herskowitz: Phys. Fluids $\underline{25}$ (1982) 2135.

63) Ch. Hollenstein, M. Guyot, and E.S. Weibel: Phys. Rev. Lett. $\underline{45}(1980) 2110$. 


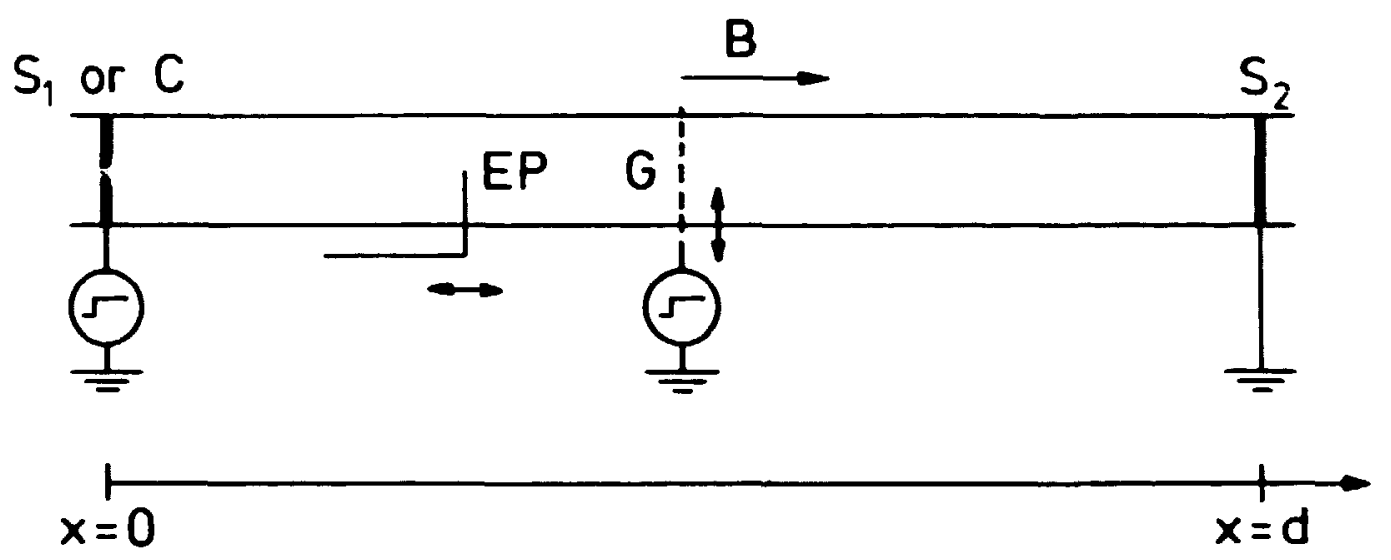

Fig. 1 Experimental set-up. In double-ended operation the plasma is generated at the two sources $s_{1}$ and $s_{2}$; in single-ended operation $S_{1}$ is replaced by the cold collector $c$. 

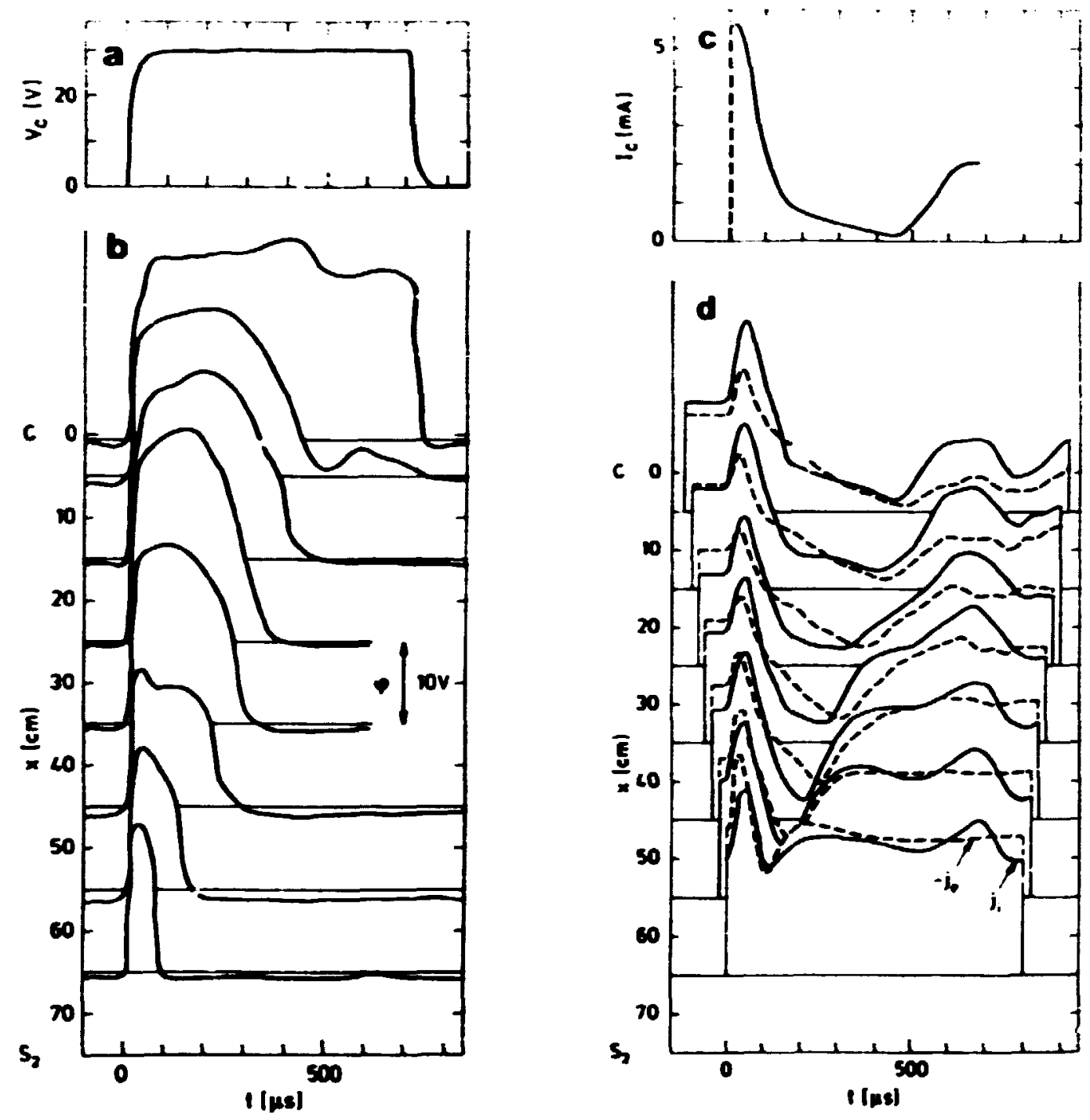

Fig. 2 Plasma response when a potential pulse is applied to the collector, $d=75 \mathrm{~cm}$. a) Applied pulse, $v_{c}$. b) Temporal evolutions of the plasma potential at different positions. c) Collector current measured through a 22 \& resistor. d) Temporal evolutions of the $i$ on $\left(j_{i}\right)$ and electron saturation current $\left(j_{e}\right)$ at different positions. 


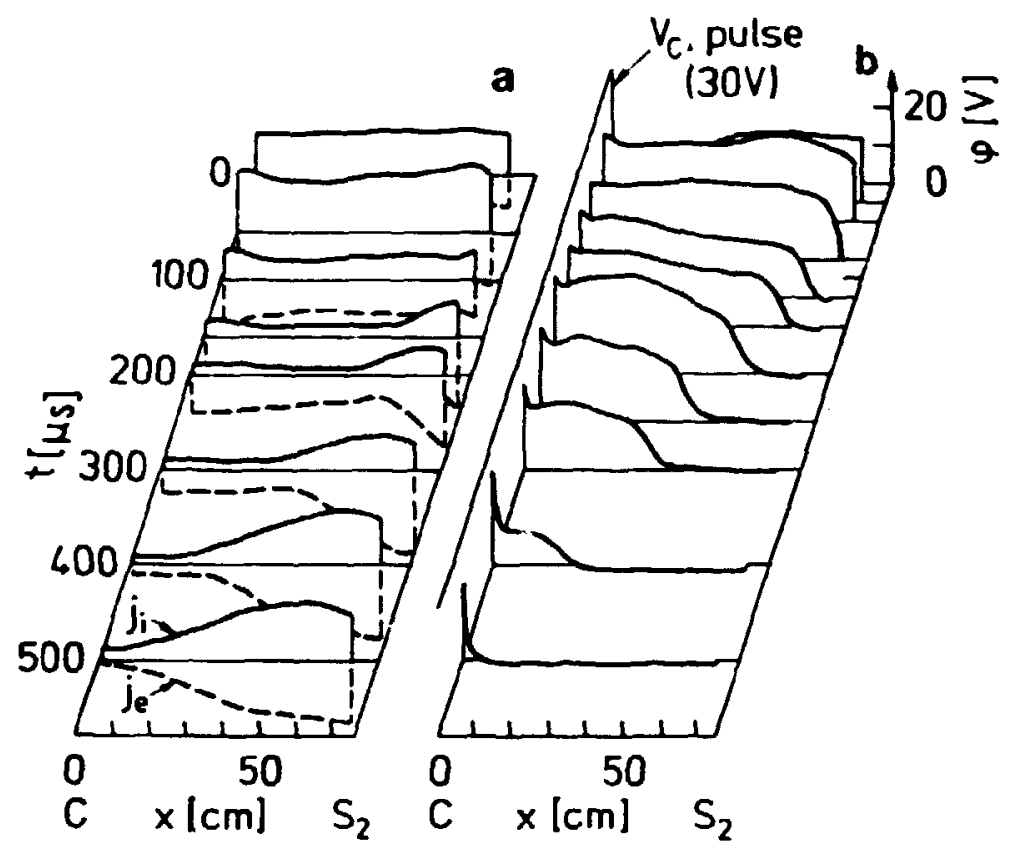

Fig. 3 Spatial evolutions of the saturation currents $j_{i}$ and $j_{e}$ (a) and the plasma potential (b) at different times after the pulse is applied. Same conditions as in Fig. 2 .

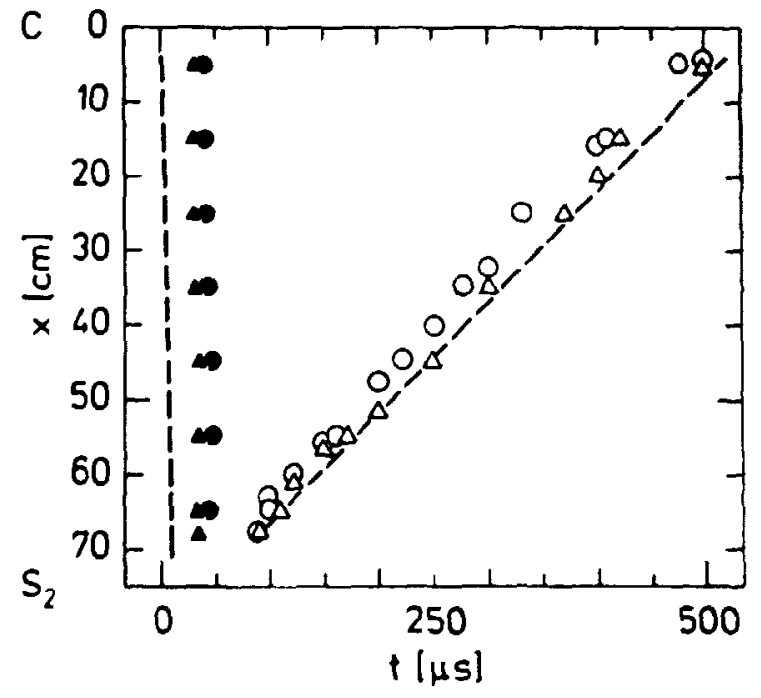

Fig. 4 Trajectory of the low potential edge of the double layer (broken line), the maxima of the saturation currents (full symbols $\Delta: j_{e}, \bullet: j_{j}$ ) and the minima of the saturation currents (open symbols $\Delta: j_{e}, 0: j_{i}$ ) obtained from Figs. 2 and 3. 

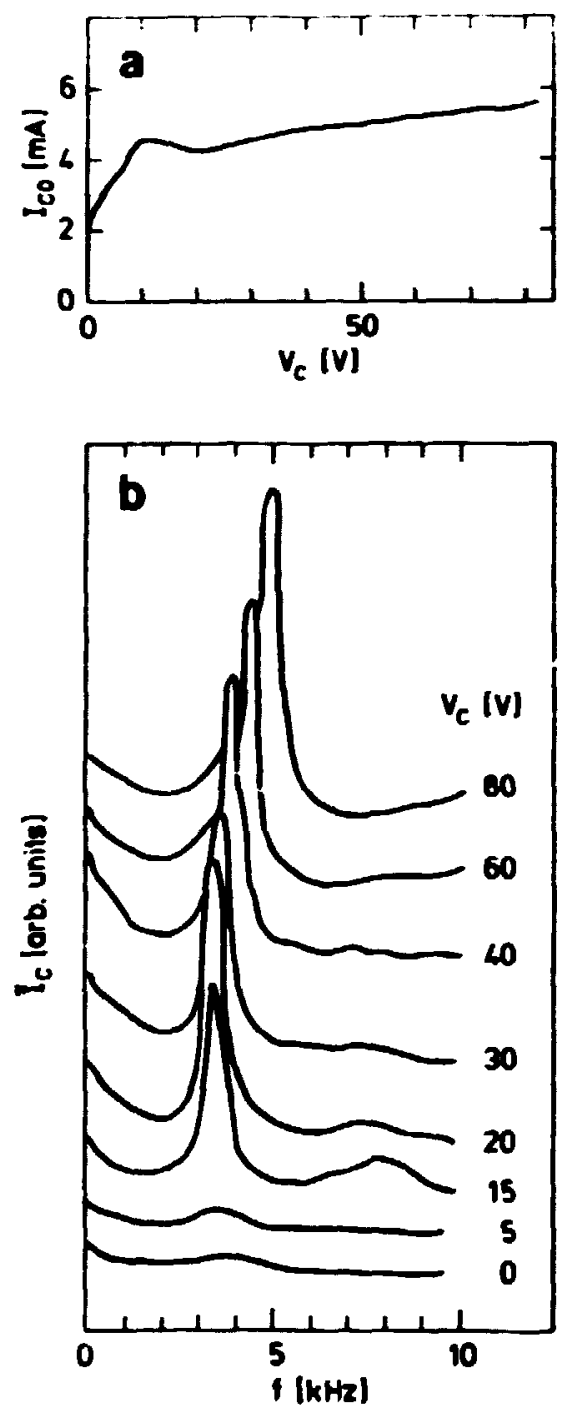

Fig. 5 The A.c. component of the collector current $I_{\text {co }}$ versus the applied potential $v_{c}$. D) Frequency spectrum of the oscillating part of the collector current $T_{f}$ for varicus applied potentials $V_{c} \cdot d=30 \mathrm{~cm}$. The currents are measured through a $22 \&$ resistor. 


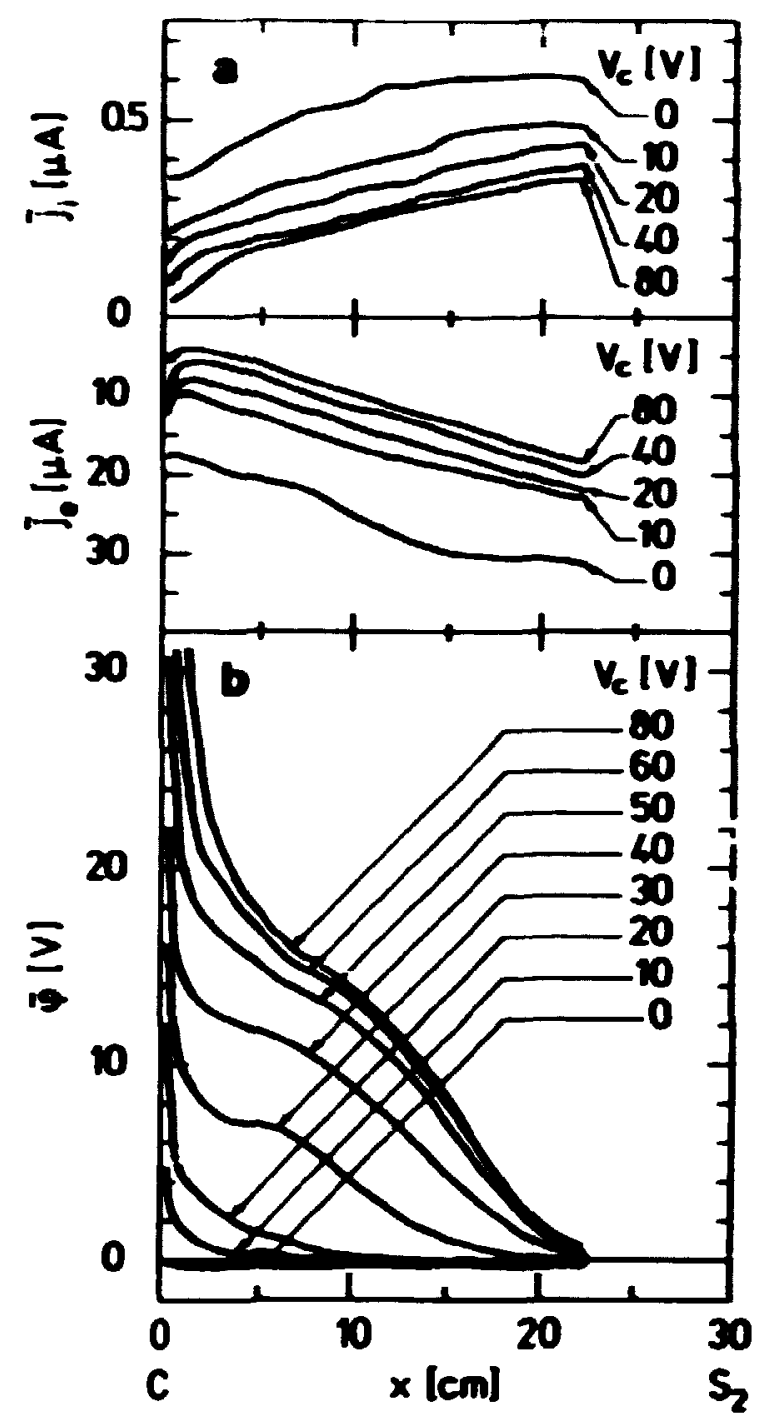

Pig. 6 Spatial profiles of the tine averaged saturation current $\hat{J}_{j}$ and $\hat{J}_{e}$ (a) and plasma potential $(b)$ for various collector potentials $v_{c} \cdot d=30 \mathrm{~cm}$. 

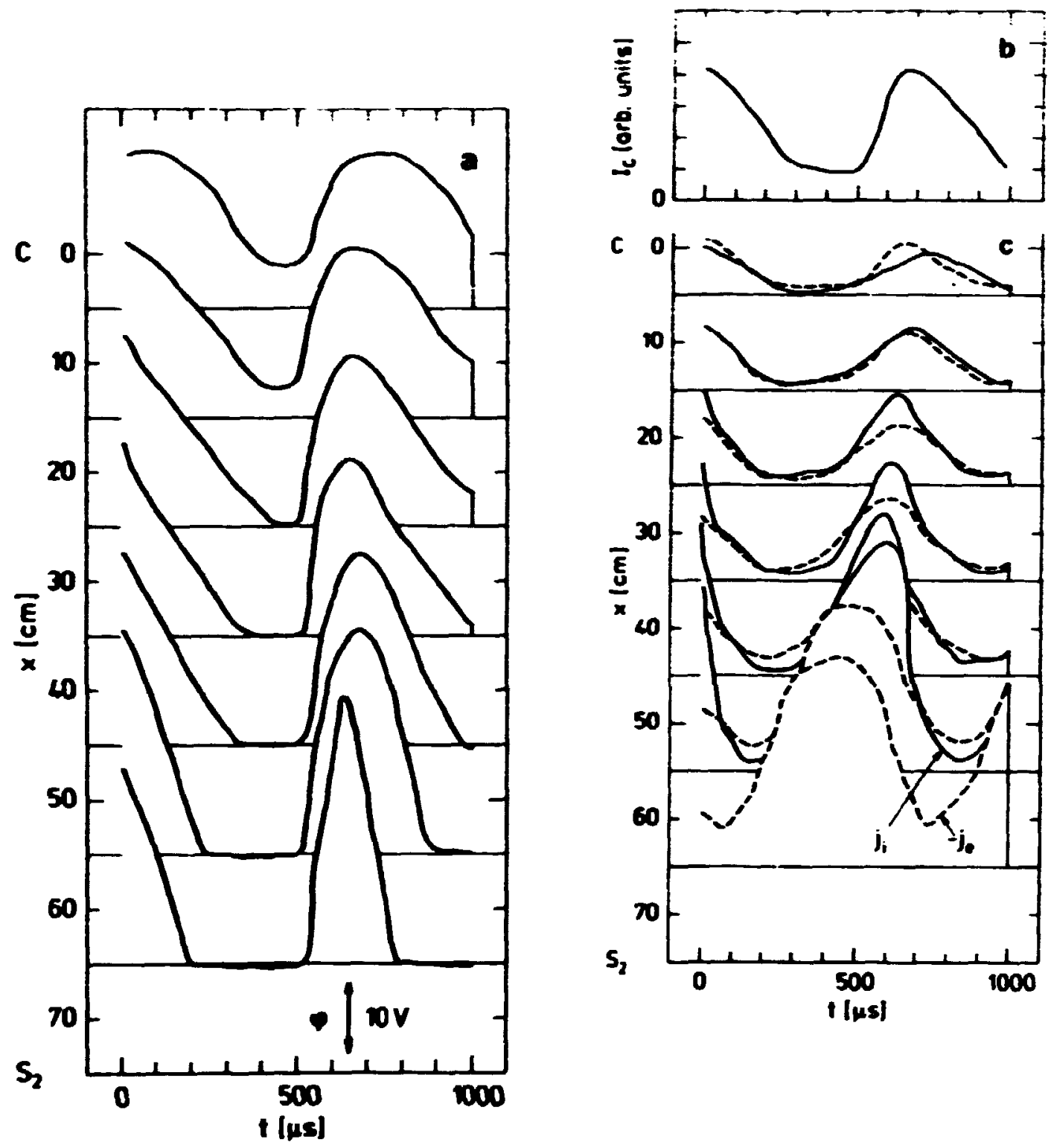

Fig. 7 Plasma response when a d.c. bias $V_{c}=72 V$ is applied to the collector. a) Temporal evolution of the plasma potential at different positions. b) The collector current, $\left.I_{c} .=\right)$ Temporal evolutions of the saturations currents $j_{i}$ and $j_{e}$ at different positions. $d=75 \mathrm{~cm}$. 


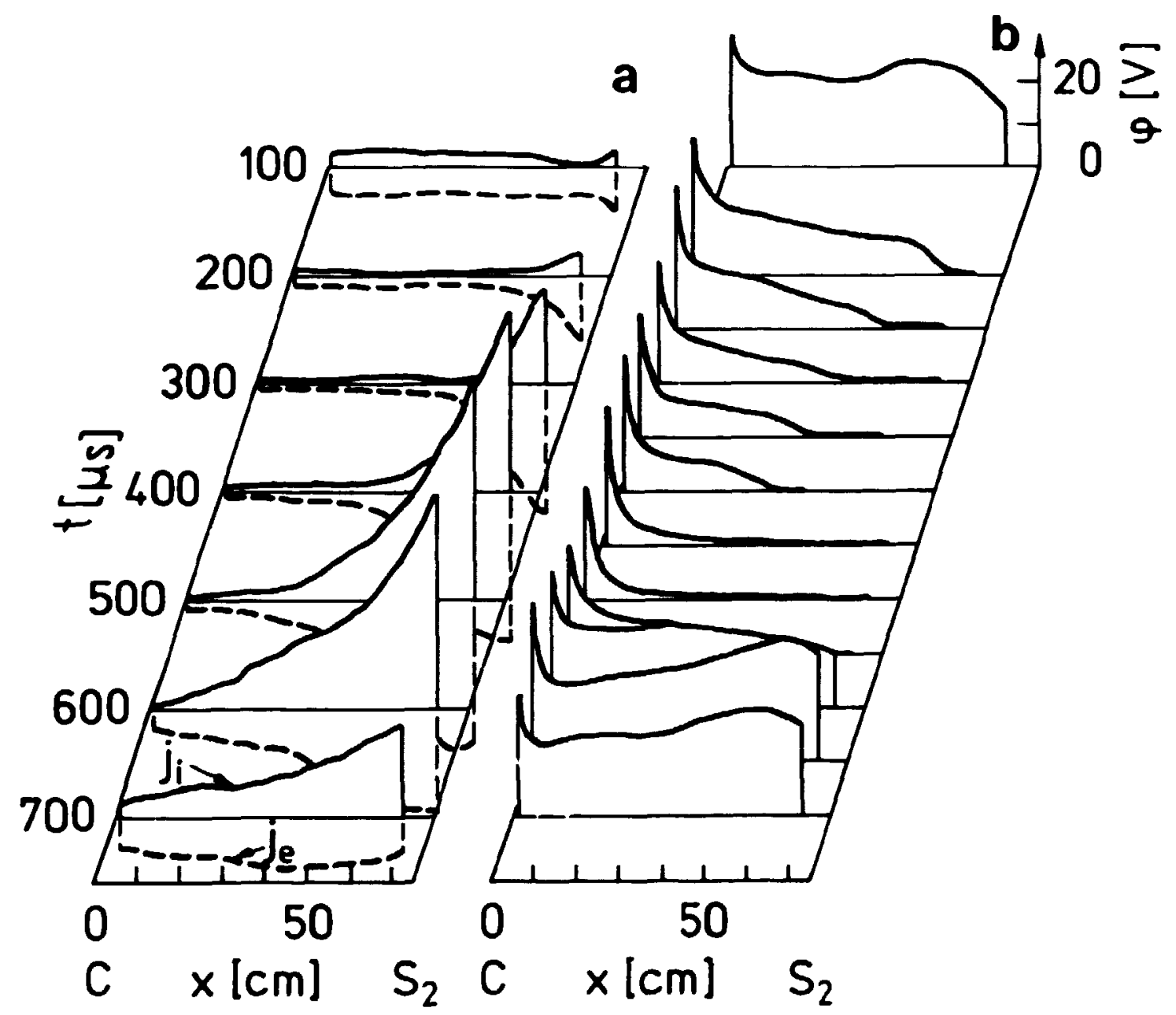

Fig. 8 Spatial evolutions of the saturation currents $j_{e}$ and $j_{i}$ (a) and the plasma potential $\phi$ (b) at different times within one period of the oscillations in $I_{c}$ (Fig. $7 b$ ). same conditions as in Fig. 7 . 


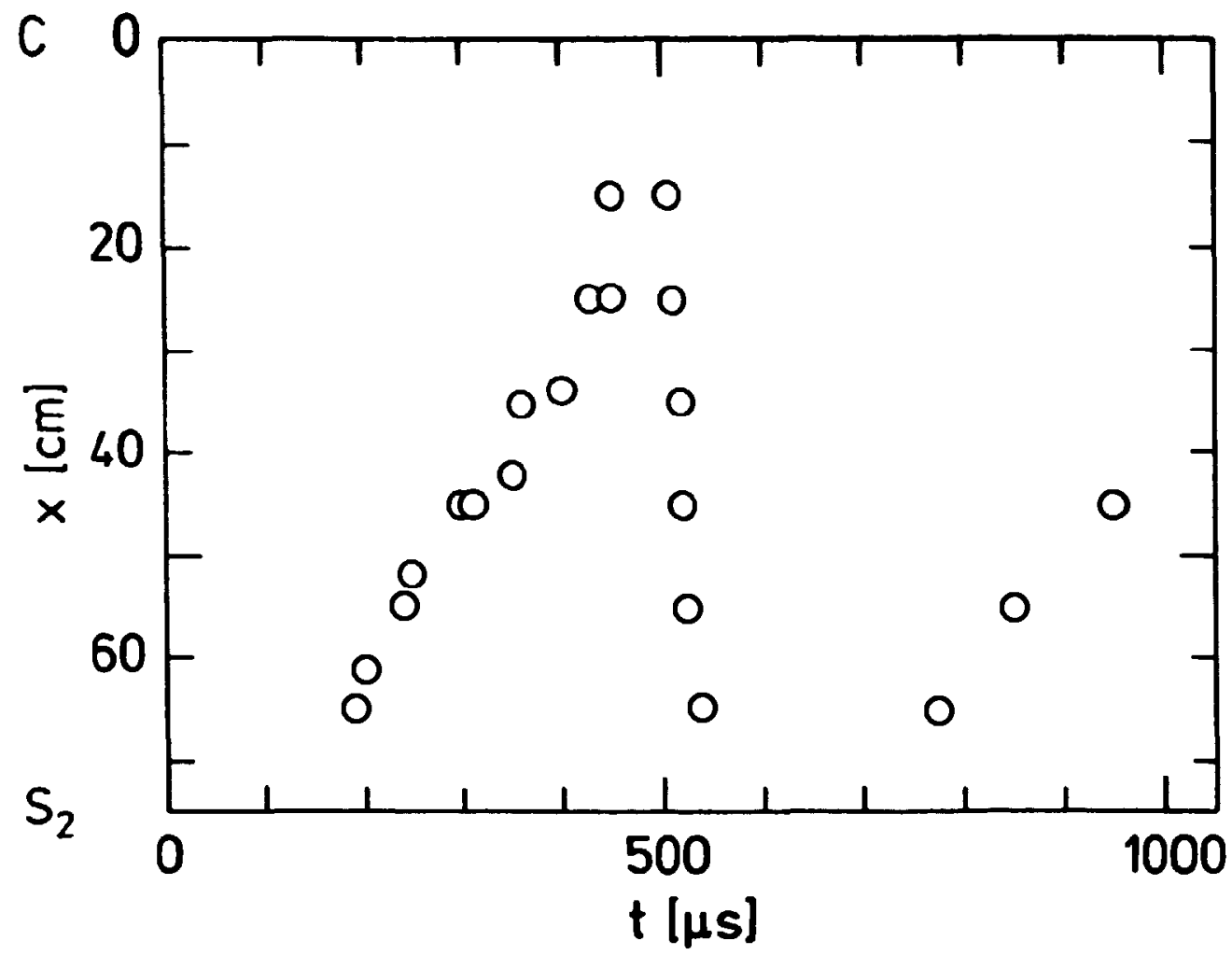

Fig. 9 Trajectory of the low potential edge of the double layer, obtained from Figs. $7 a$ and $8 b$.

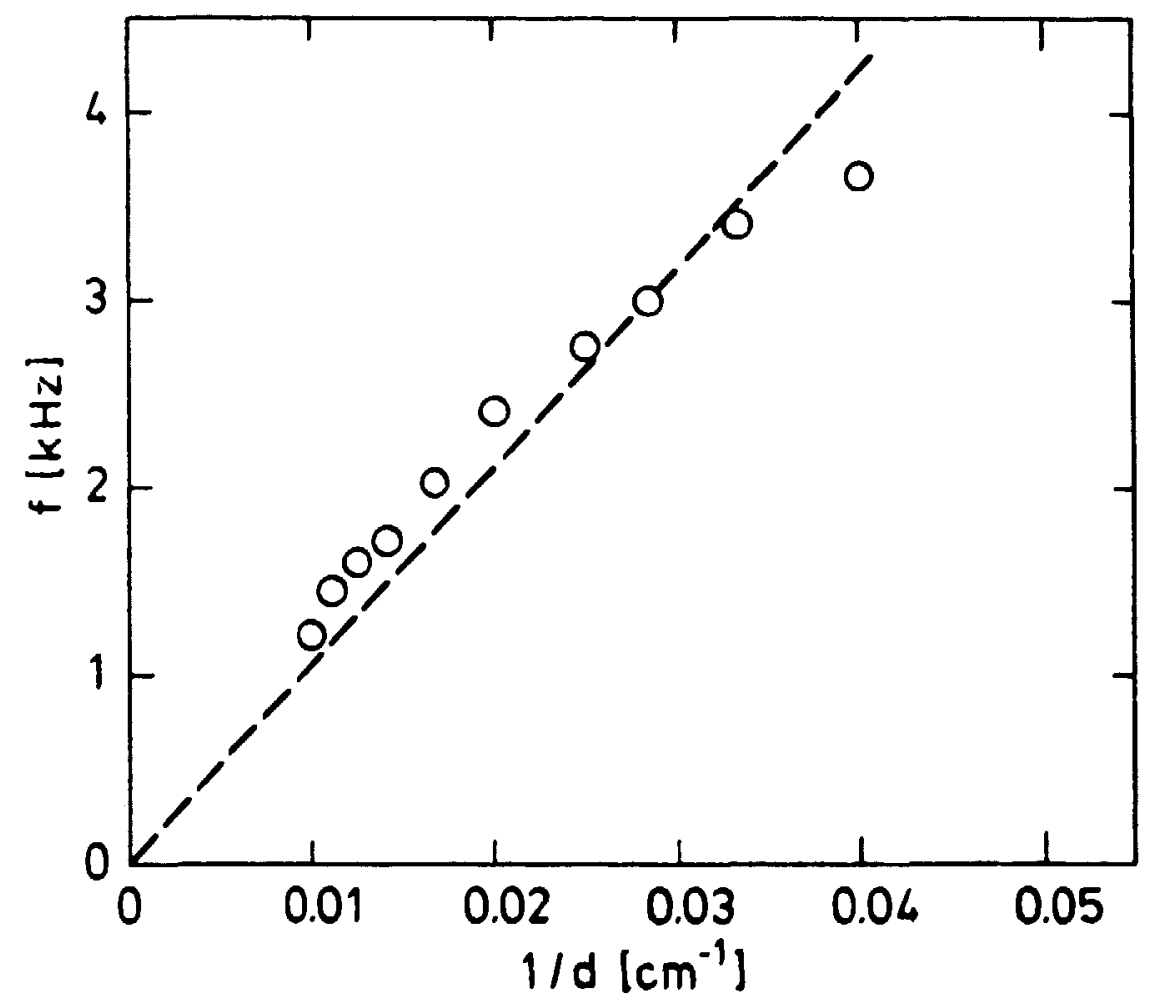

Fig. 10 Frequency $f$ of the oscillations versus the inverse length $1 / \mathrm{d}$ of the plasma column $\mathrm{v}_{\mathrm{C}}=30 \mathrm{~V}$. 


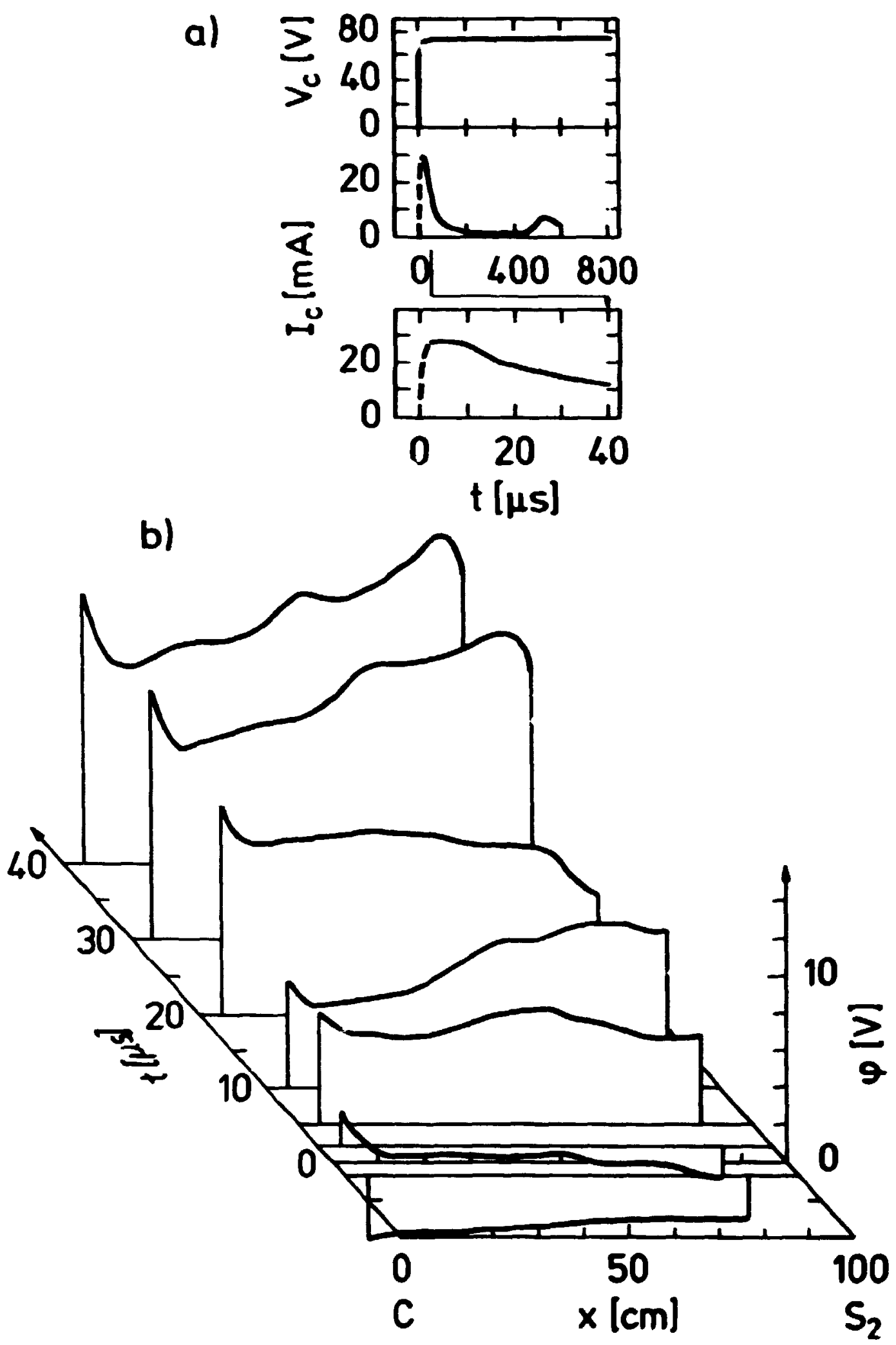

Fig. 11 a) Potential $V_{c}$ applied to the collector and the collector current $I_{c}$ on different time scales. b) The initial evolution of the plasma potential . d $=100 \mathrm{~cm}$. 


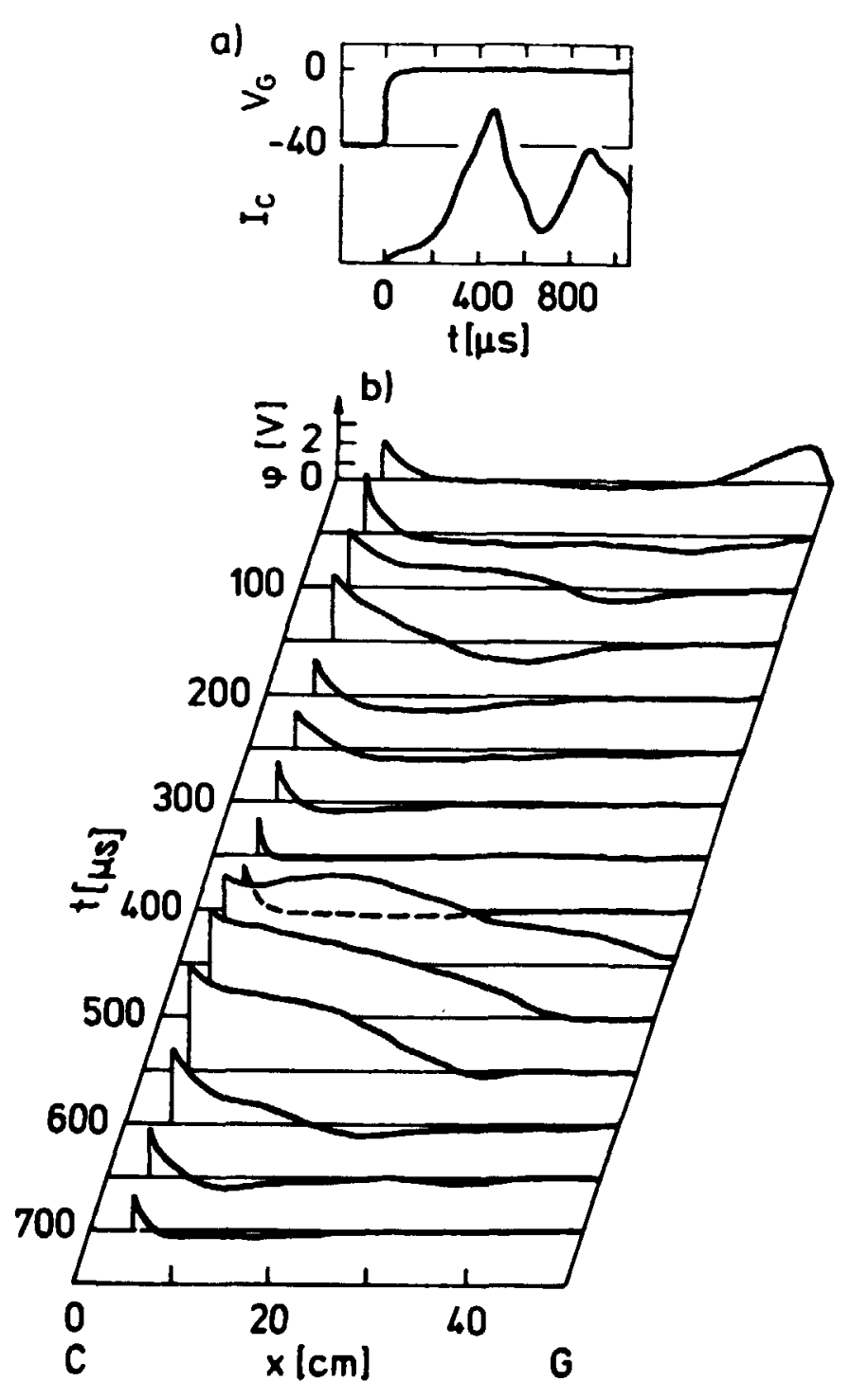

Fig. 12 Plasma expansion. a) The potential $V_{G}$ applied to the grid at $x=50 \mathrm{~cm}$ and the collector current $I_{c}$. b) Evolution of the plasma potential in the region between the grid and the collector. c) simultaneous evolution of the saturation currents $j_{i}$ and $j_{e}$. $d=100 \mathrm{~cm}$ and $v_{c}=50 \mathrm{~V}$. 


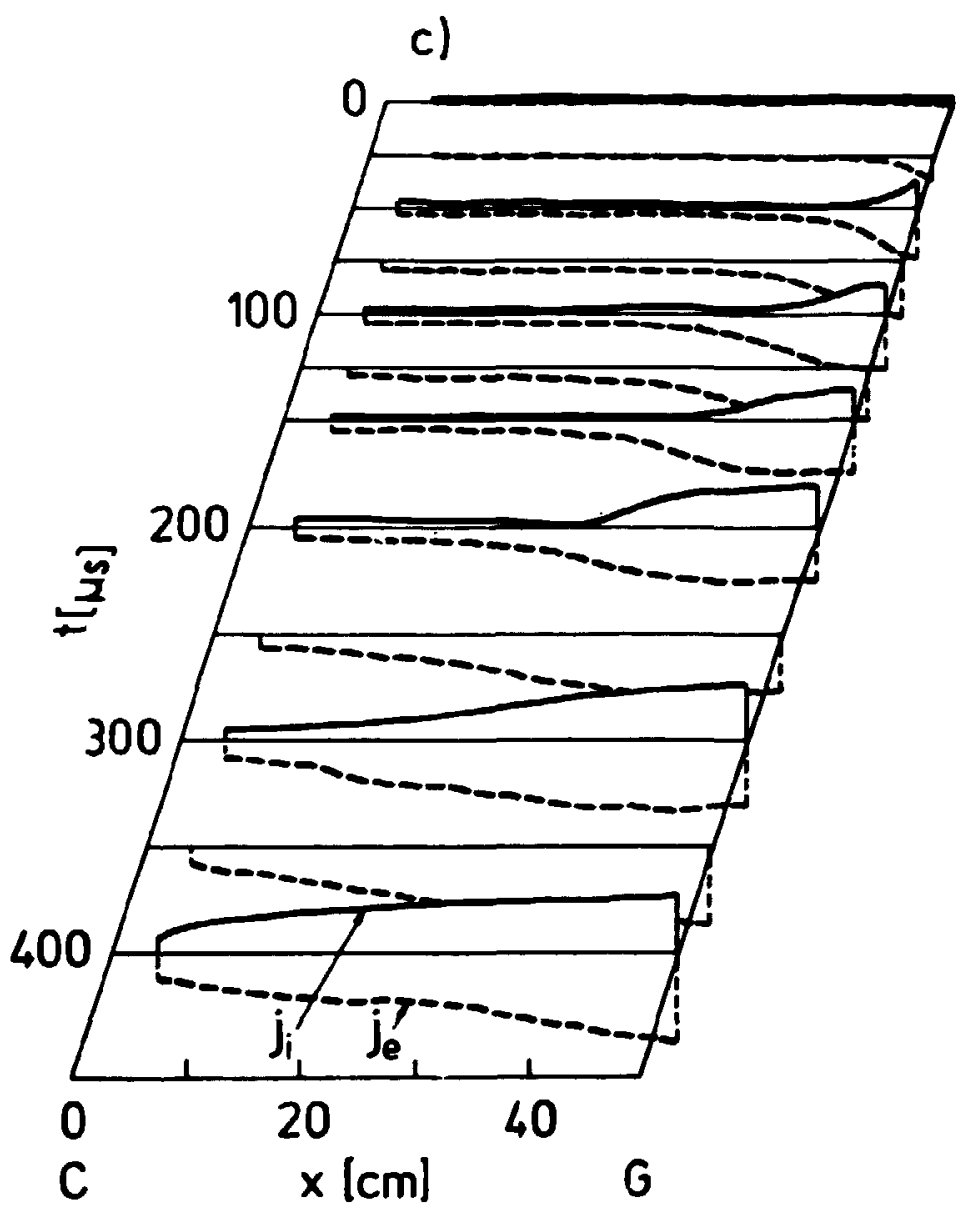

Fig. 12 Continued. 


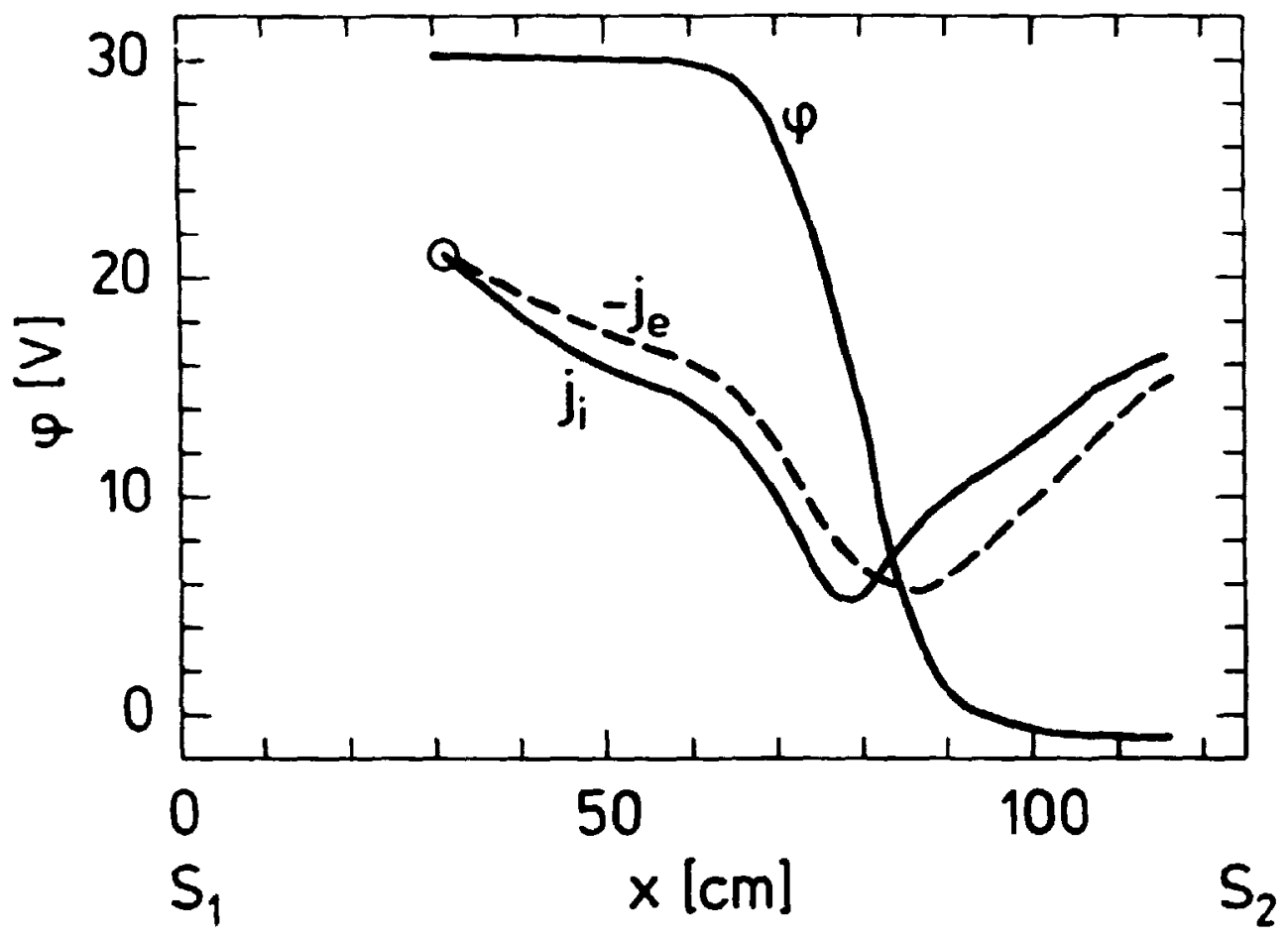

Fig. 13 The stationary double layer formed when the machine is operated in double-ended mode $w i$ th the source $S_{1}$ biased, $V_{S 1}=30 \mathrm{~V}$. $d=125 \mathrm{~cm}$. Time averaged profiles of the plasma potential and the sautration currents $j_{i}$ and $j e$. The saturation currents are plotted in arbitrary units and normalized to the same value at the point marked with the circle. 

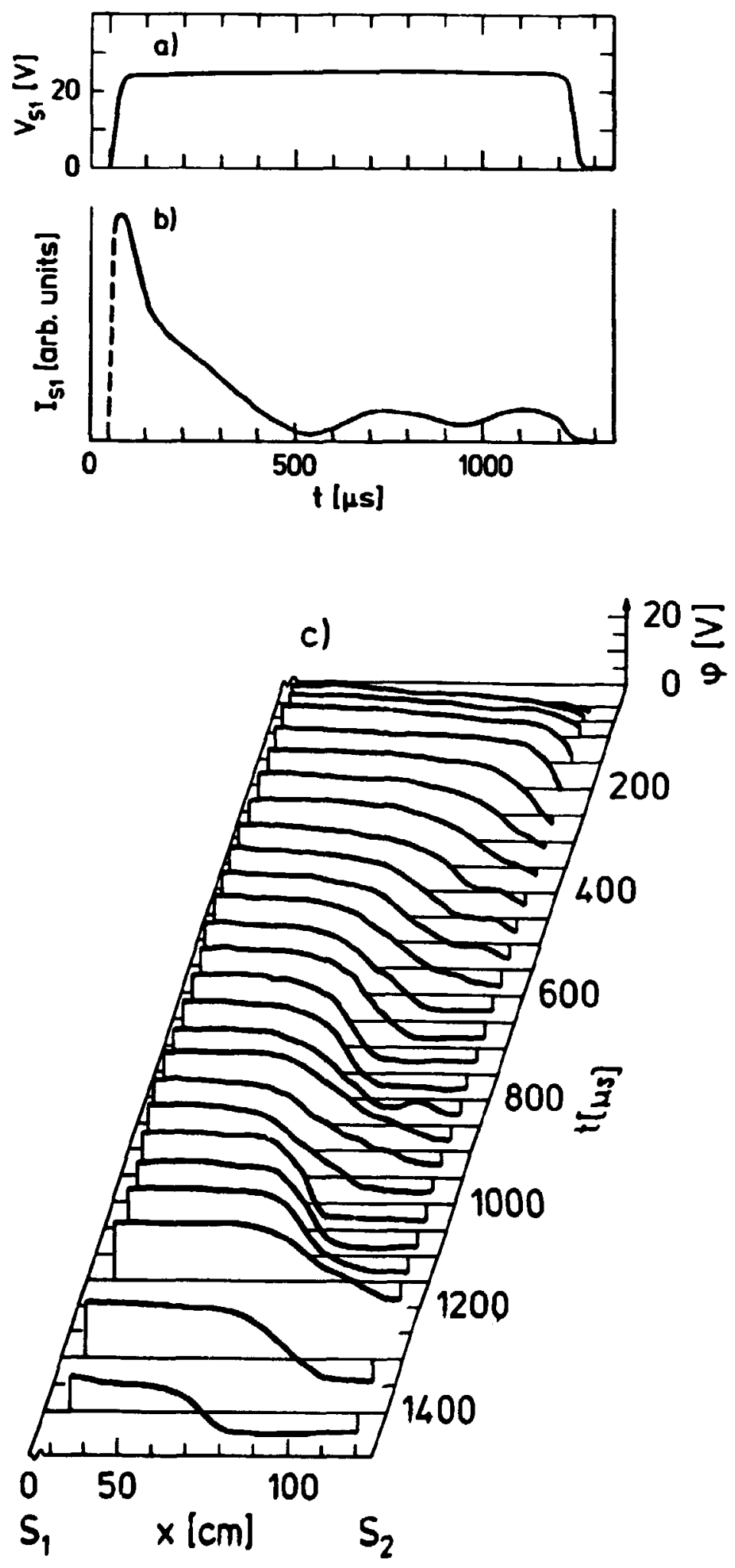

Fig. 14 Development of the double layer when the bias on $s_{1}$ is pulsed. a) Applied potential $V_{S_{1}}=25 \mathrm{~V}$. b) Current to $s_{1}$. Is1. c) Spatial evolutions of the plasma potential $\phi$. 


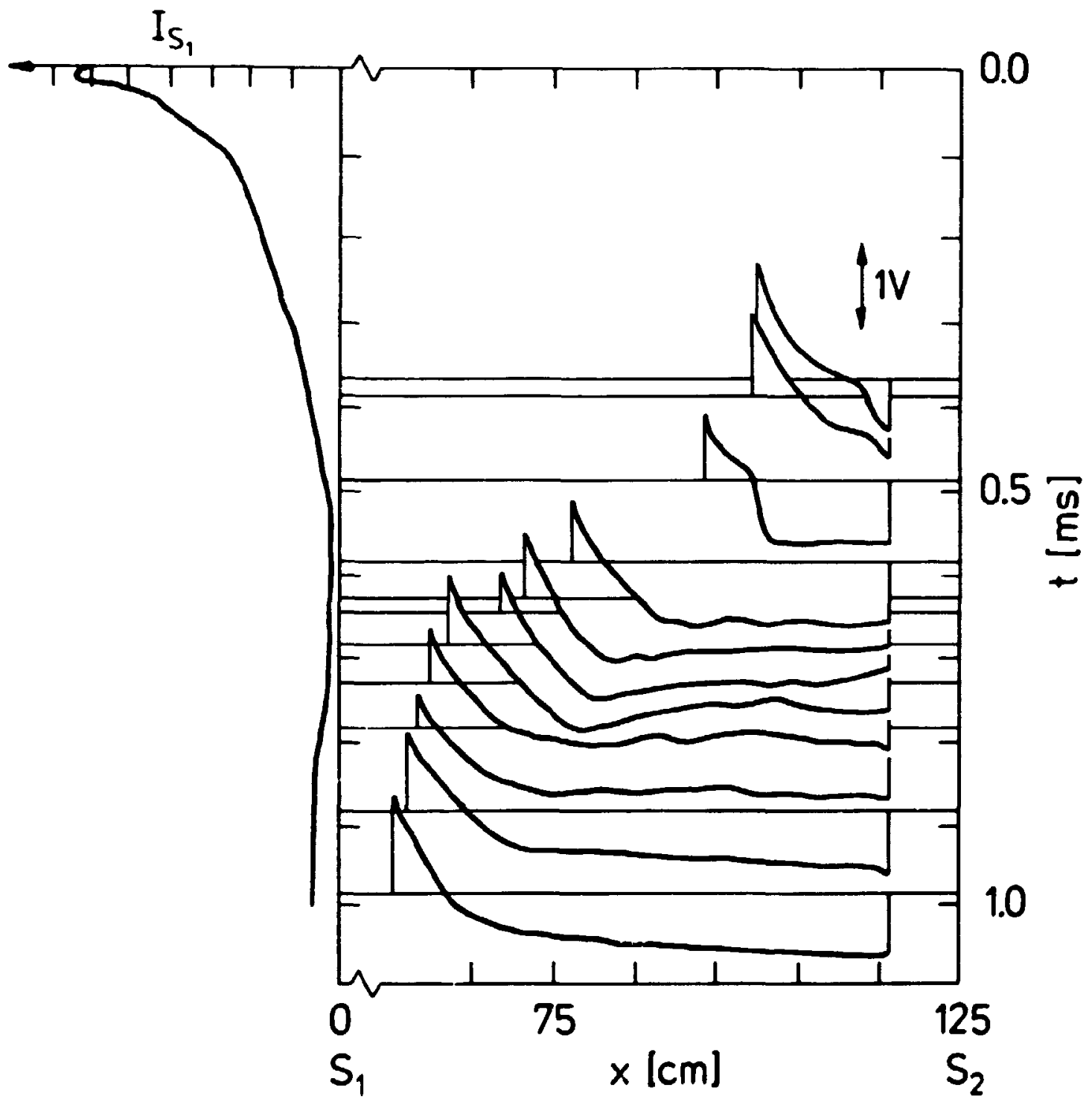

F1g. 15 Detalled evolution of the low potential side of the double layer and the varlation of the current $I_{S 1}$ when a pulse is applied to $s_{1} \cdot V_{S 1}=34 \mathrm{~V}$. Note, not the same conditions as in Fig. 14. 



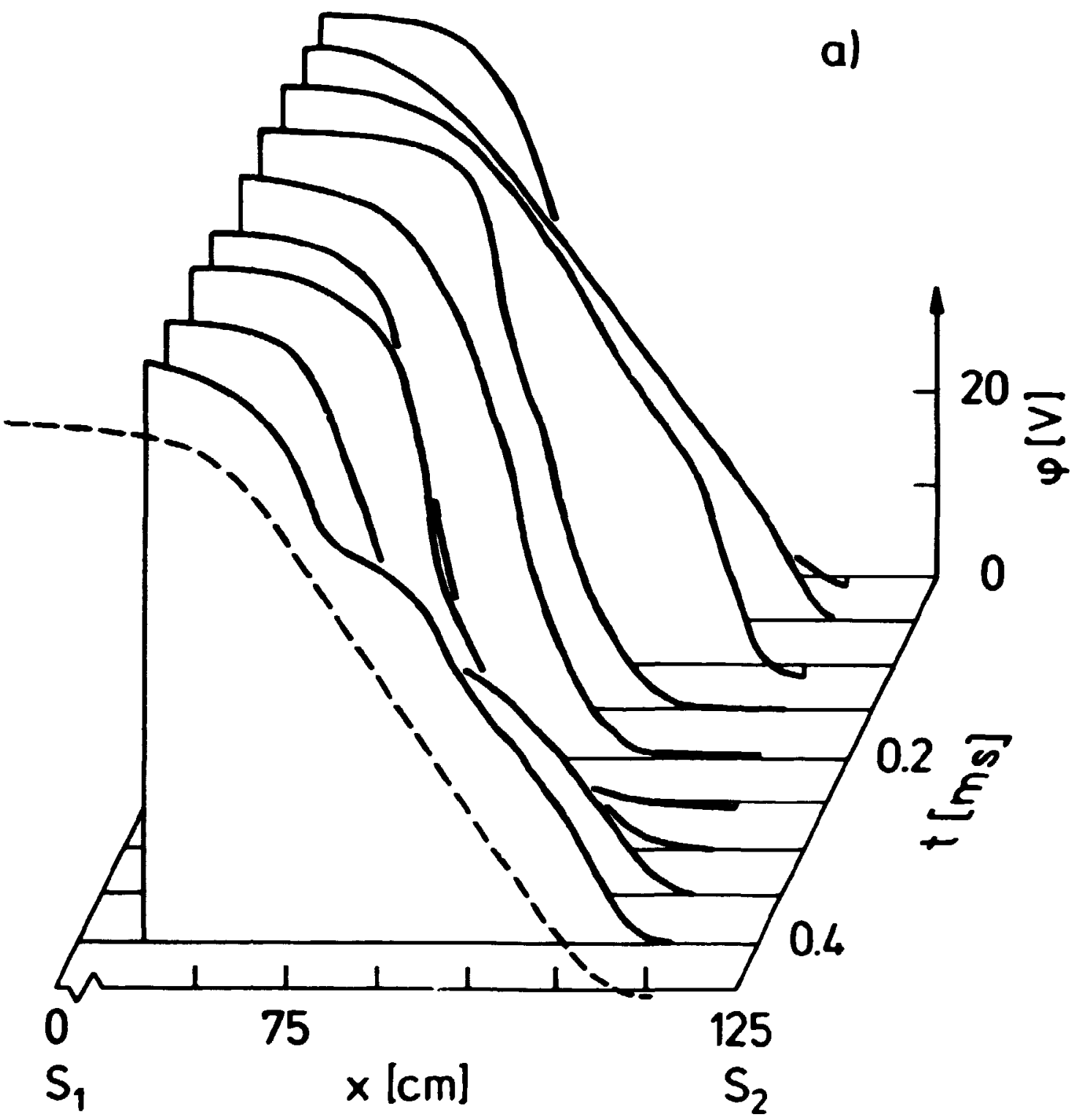

Pig. 16 a) Evo.-ution of the double layer profile within one period of the oscillation in the current ISI (b). Broken curve shows the time averaged profile. VSi = $61 \mathrm{~V}$. c) Trajectory of the low potential edge of the double layer. d) Prequency $f$ of the oscillations in Is 1 versus the inverse distance $1 / L$ between $S_{2}$ and the midpoint of the time averaged double layer profile. 

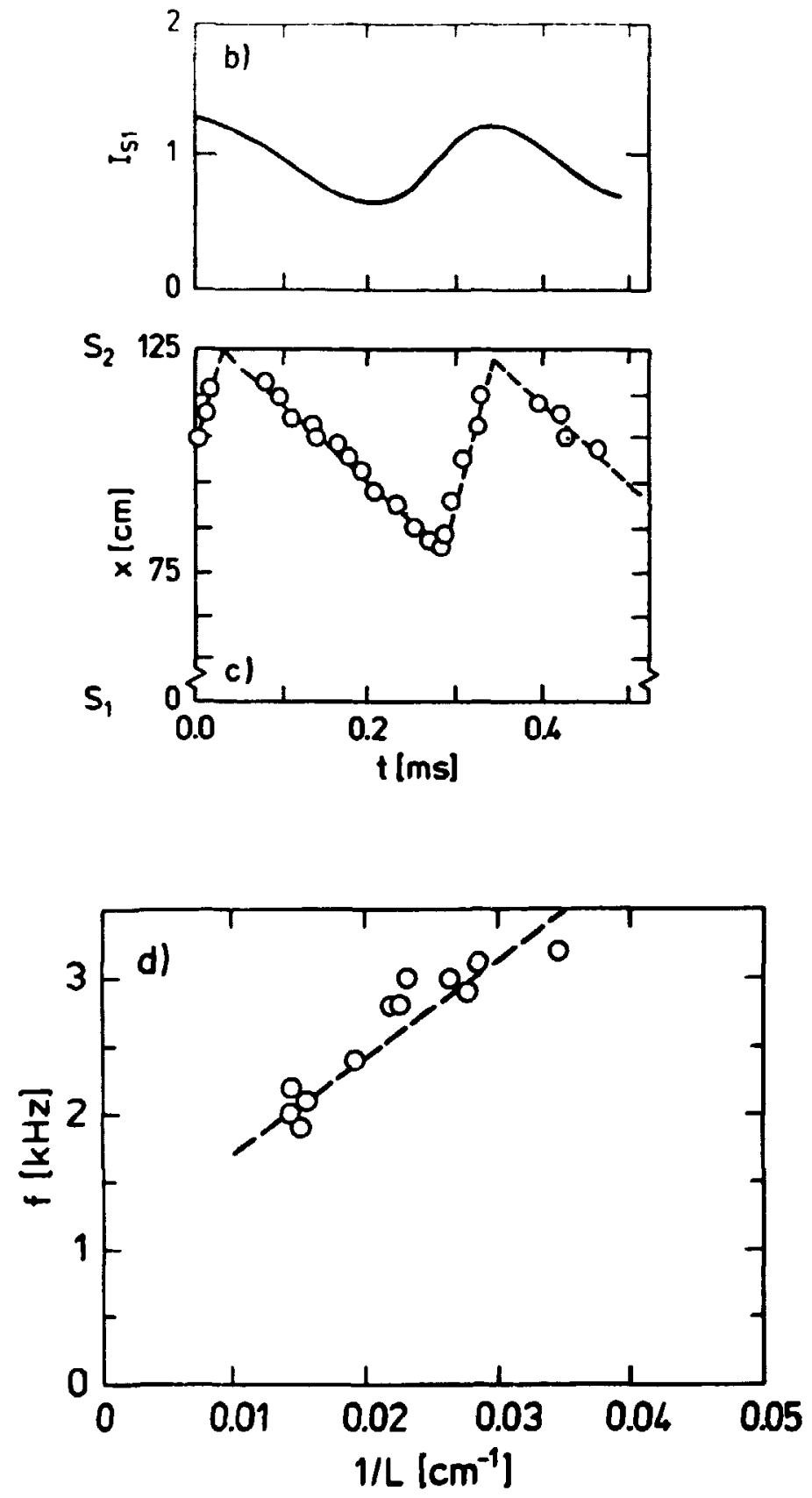

Fig. 16 Continued. 


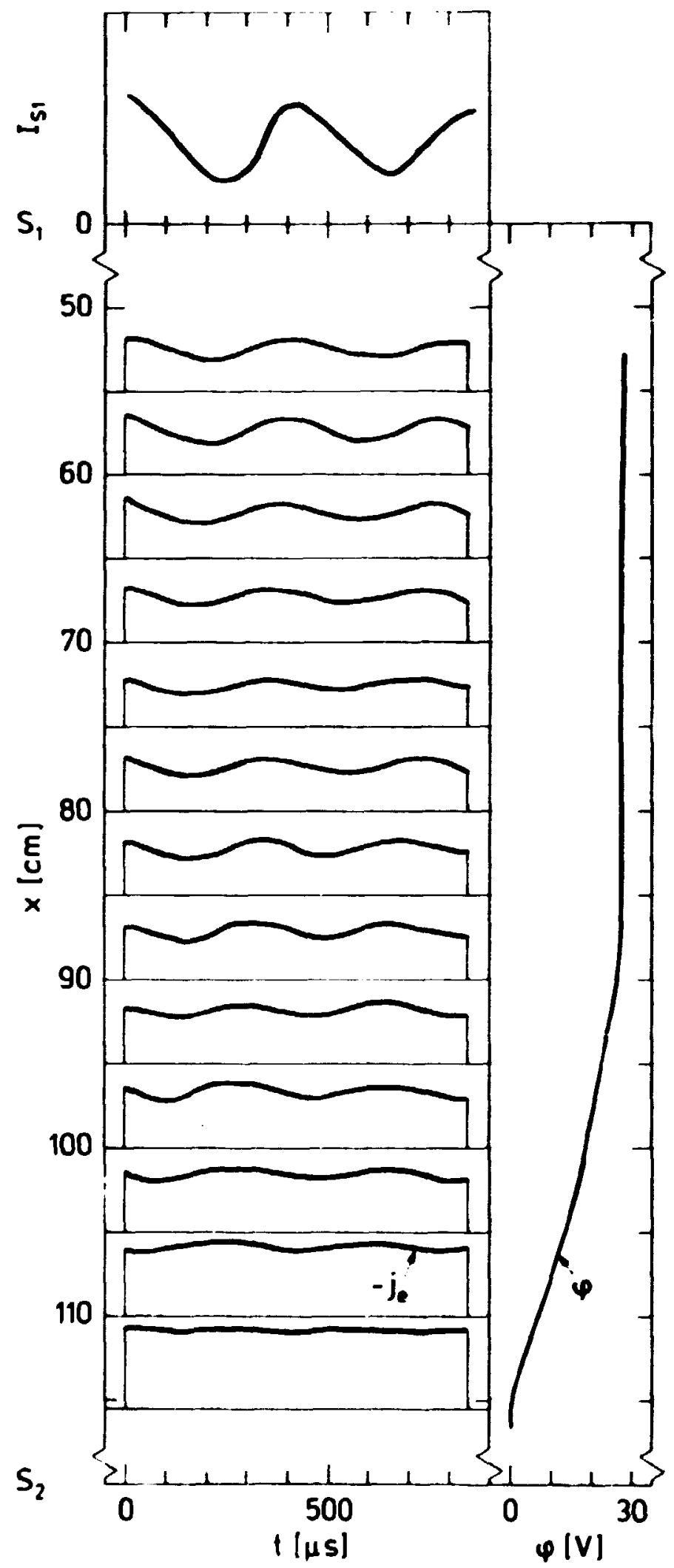

Fig. 17 Evolution of the electron saturation current je together with the current $I_{s 1}$ and the averaged double layer profile when a d.c. bias is applied to $s_{1}, v_{S 1}=$ $30 \mathrm{~V}$. 


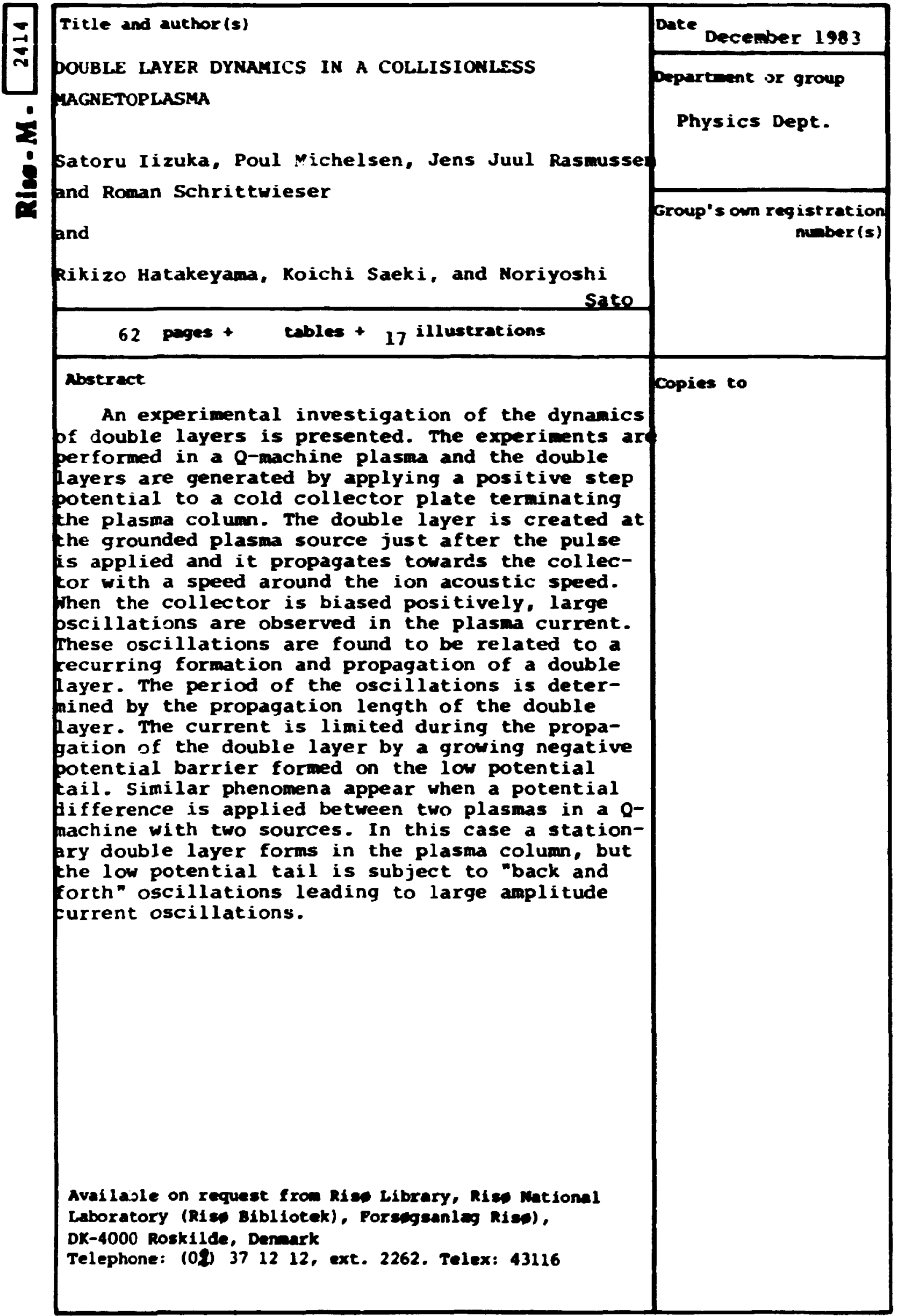

